DOE/ER/40147--10

DE92 007707

\title{
NUCLEAR INTERACTIONS OF HIGH ENERGY HEAVY IONS AND APPLICATIONS IN ASTROPHYSICS
}

\author{
Technical Progress Report
}

1 April 1991 - 31 March 1992

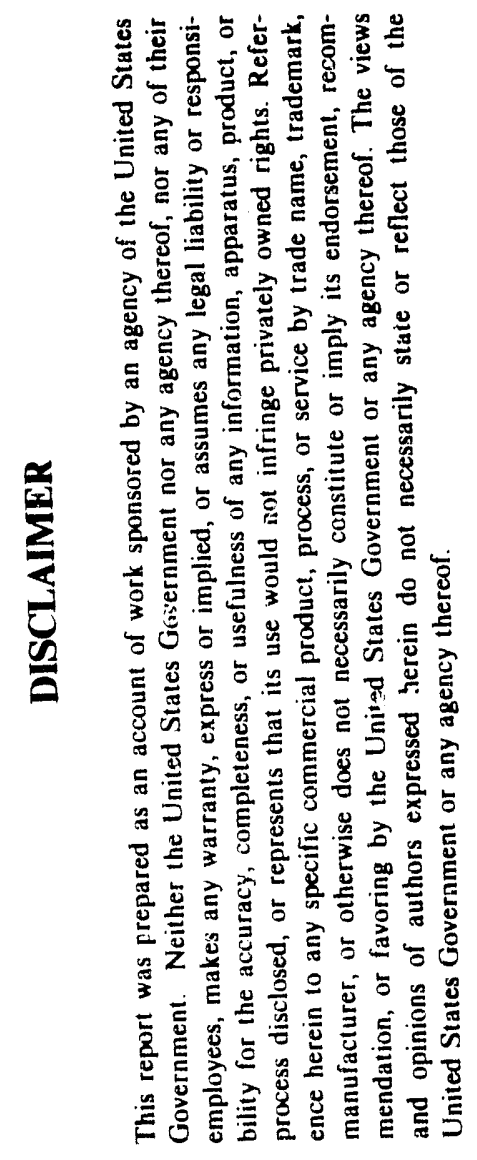

\author{
John P. Wefel \\ Department of Physics and Astronomy \\ Louisiana State University \\ Baton Rouge, LA 70803-4001 \\ 504-388-8696
}

23 January 1992

Prepared for the U.S. Department of Energy

Grant Number DE-FG05-84ER40147

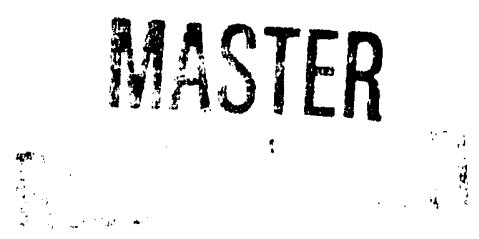

FEB 1 \& 992 , 


\title{
TECHNICAL PROGRESS REPORT
}

\author{
Grant DE-FG05-84ER40147
}

John P. Wefel, Principal Investigator

T. Gregory Guzik, Co-Investigator

\section{SUMMARY}

This past year has involved both data-taking and analysis, and significant progress has been made in the analysis of the existing datasets. In addition, we performed in a successful HISS run, using the liquid hydrogen target, to obtain 14 new datasets.

Our HISS experiment, E938H, had a full month's run at the Bevalac. Measurements were extended to $800 \mathrm{MeV} /$ nucleon and to additional beams, many of which were neutron rich species (e.g. ${ }^{22} \mathrm{Ne}$ ). Planned runs at $1.6 \mathrm{GeV} /$ nucleon were cut short when an MG set failed, and the remaining time was utilized to fill out the database at the lower energies.

Analysis of the HISS datasets has proceeded to the point that preliminary isotopic cross sections are available for ${ }^{32} \mathrm{~S}$ at $400 \mathrm{MeV} /$ nucleon and total charge changing plus elemental cross sections have been determined for many of the beam-energy points.

The low energy ${ }^{28} \mathrm{Si}$ analysis has progressed to the point that isotopic cross sections are available for the elements $\mathrm{C}$ through $\mathrm{Mg}$, and final background corrections for $\mathrm{Al}$ are underway. In addition, cross sections from the lowest energy 160 run show some surprising behavior. Rechecking of these results is the next step.

During the past year, the review paper by J. P. Wefel has appeared in print:

"The Composition of the Cosmic Riays: An Update," John P. Wefel, in Cosmic Rays, Supernovae and the Interstellar Medium, eds. M. M. Shapiro, R. Silberberg and J. P. Wefel, (Dordrecht, The Netherlands; 1991; Kluwer Academic Publ.), pp. 29-55.

and several contributed papers were given at an international conference:

"Low Energy ( $E<360 \mathrm{MeV} /$ nucleon) Fragmentation Cross Sections for Use in GCR Propagation Calculations," H.J. Crawford, J. Engelage, T.G. Guzik, M. Hof, M. Hollier, J. Isbert, P.J. Lindstrom, K.D. Mathis, J.W. Mitchell, J. Neuhous, W. Schimmerling, M. Simon, J.P. Wefel, D. Williams, 22nd ICR Conference Papers, (Dublin, Ireland, 1991, Institute for Advanced Studies), 2, 284.

"Charge Change Total Cross Section Measurements of Heavy Nuclide Fragmentation at LBL HISS Facility," C.-X. Chen, S. Costa, H.J. Crawford, J. Engelage, P. Ferrando, L. Greiner, T.G. Guzik, F.C. Jones, C.N. Knott, S. Ko, C. Kuo, P.J. Lindstrom, U. Lynen, J. Mazotta, J.W. Mitchell, W.F.J. Mueller, D. Olson, R. Potenza, A. Soutoul, T.J.M. Symons, O. Testard, C.E. Tull, C.J. Waddington, W.R. Webber, J.P. Wefel, H.H. Wieman, 22nd ICR Conference Papers, (Dublin, Ireland, 1991, Institute for Advanced Studies), 2, 296.

"Isotopic Production Cross Sections from Projectile Fragmentation of Relativistic Heavy Ions," C.E. Tull, C.-X. Chen, S. Costa, H.J. Crawford, J. Engelage, P. Ferrando, L. Greiner, T.G. Guzik, F.C. Jones, C.N. Knott, S. Ko, C. Kuo, P.J. Lindstrom, U. Lynen, J. Mazotta, J.W. Mitchell, W.F.J. Mueller, D. Olson, R. 
Potenza, A. Soutoul, T.J.M. Symons, O. Testard, C.J. Waddington, W.R. Webber, J.P. Wefel, H.H. Wieman, 22nd ICR Conference Papers, (Dublin, Ireland, 1991, Institute for Advanced Studies), 2, 300.

"Elemental Production Cross Sections from Neon to Nickel," C.N. Knott, C.-X. Chen, S. Costa, H.J. Crawford, J. Engelage, P. Ferrando, L. Greiner, T.G. Guzik, F.C. Jones, S. Ko, C. Kuo, P.J. Lindstrom, U. Lynen, J. Mazotta, J.W. Mitchell, W.F.J. Mueller, D. Olson, R. Potenza, A. Soutoul, T.J.M. Symons, O. Testard, C.E. Tull, C.J. Waddington, W.R. Webber, J.P. Wefel, H.H. Wieman, 22nd ICR Conference Papers, (Dublin, Ireland, 1991, Institute for Advanced Studies), 2, 292.

"A Heavy Ion Spectrometer System used for the Measurement of Projectile Fragmentation of Relativistic Heavy Ions," J. Engelage, S. Albergo, C.-X. Chen, S. Costa, H.J. Crawford, P. Ferrando, L. Greiner, T.G. Guzik, F.C. Jones, C.N. Knott, S. Ko, C. Kuo, P.J. Lindstrom, U. Lynen, J. Mazotta, J.W. Mitchell, W.F.J. Mueller, D. Olson, R. Potenza, A. Soutoul, T.J.M. Symons, O. Testard, C.E. Tull, C. Tuve, C.J. Waddington, W.R. Webber, J.P. Wefel, H.H. Wieman, 22nd ICR Conference Papers, (Dublin, Ireland, 1991, Institute for Advanced Studies), 2, 531.

Several papers are scheduled for presentation at the spring APS meeting:

"Elemental Production Cross Sections from Neon to Nickel: The Transport Collaboration," C.N. Knott et al.

"Charge Changing Total Cross Sections of Heavy Ions on Liquid Hydrogen Target," C.-X. Chen et al.

"Isotopic Production Cross Sections from Projectile Fragmentation of ${ }^{32} \mathrm{~S}$ in a Liquid Hydrogen Target," C.E. Tull et al.

and an abstract has been submitted to the COSPAR conference to the held in Washington, DC in August, 1992,

"A Program to Measure New Energetic Particle Nuclear Interaction Cross Sections," T. Gregory Guzik et al.

Reprint:s/preprints of the available papers are attached.

\section{PROJECT OVERVIEW}

This program was established for the purpose of studying projectile fragmentation; (1) as a function of energy, focusing first on the intermediate energy region, $<1 \mathrm{GeV} /$ nucleon, where there have been few previous measurements and no systematic studies, and (2) as a function of projectile mass, starting with light beams and proceeding to species as heavy as nickel (and possibly beyond). The intermediate energy region is important as the transition between the lower energy data, where the interaction appears to be dominated by collective effects and the decay of excited nuclei, and the highest energy results, where nucleon-nucleon interactions are fundamental, 'limiting fragmentation' applies, and the nucleus may well break-up before any de-excitation. The mass dependence of projectile fragmentation is largely unknown since most detailed work has involved light ion beams. Nuclear structure effects, for example, may well be quite prominent for heavier beams. Furthermore, the nuclear excitation functions for the production of different fragment isotopes have immediate application to the astrophysical interpretation of existing isotopic datasets obtained from balloon and satellite measurements of galactic cosmic rays. 


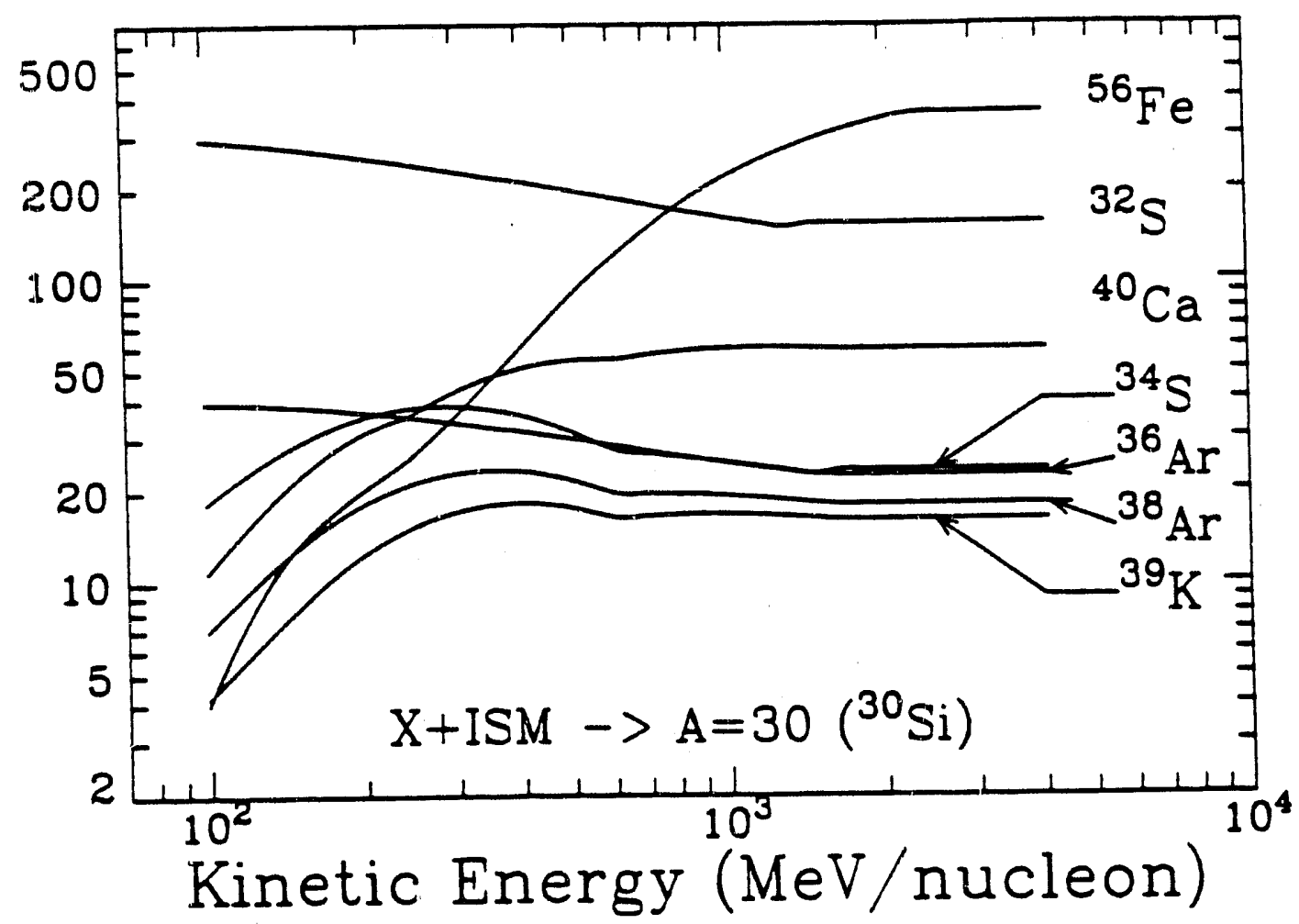

Figure 1. The relative contribution of different isotopes to the production of secondary ${ }^{30} \mathrm{Si}$ during cosmic ray propagation in the Interstellar Medium (ISM).

One astrophysical rationale for this program is illustrated in Figure 1. The isotope ${ }^{30} \mathrm{Si}$ has been found to be in excess in the cosmic rays. Some of the ${ }^{30} \mathrm{Si}$ are primary while some are secondary from nuclear fragmentation in the interstellar medium. Determining the secondary component requires measurements of the ${ }^{30} \mathrm{Si}$ production cross section from "beams" of ${ }^{56} \mathrm{Fe},{ }^{32} \mathrm{~S}$, ${ }^{40} \mathrm{Ca},{ }^{36} \mathrm{Ar},{ }^{34} \mathrm{~S},{ }^{38} \mathrm{Ar}$ and ${ }^{39} \mathrm{~K}$, approximately in order of importance above $1 \mathrm{GeV} /$ nucleon. At lower energies, the relative contributions vary with energy, requiring, for best results, knowledge of the energy dependent excitation function.

The cross sections that we are measuring also have practical applications. When the President announced his intention for this country to proceed in space to a lunar base and a Mars mission, one of the crucial questions was "how much shielding is needed." This is an area of DOE, as well as NASA and DDD, interest. Answering the shielding question requires detailed knowledge of the break-up of heavy ions, at all energies, and our databank can help to provide results for the shielding analyses. We have already received requests for the cross section values, as soon as the analysis is completed.

This project started with measurements at the B40 facility at the LBL Bevalac and has progressed to experiments using the HISS facility. The reasons for beginning with inclusive studies at intermediate energy in B40 were four-fold: (1) the intermediate energy region had been little explored, and might yield new discoveries, (2) the experimental apparatus was available and a small size collaboration could set-up and perform experiments, (3) the astrophysical applications were direct, and (4) the HISS facility already had a large experimental program. The B40 experiments have provided new information, as well as an opportunity for new detector development, and have served as an entry point to become involved in the more extensive collaborations needed to perform a HISS experiment. 
The LSU group has been a member of three separate collaborations for the work covered under this grant. For the lower energy B40 experiments, the collaboration involves researchers at Berkeley (LBL, UCBSSL, Donner), led by H. J. Crawford and including J. Engelage, P. J. Lindstrom and W. Schimmerling, and at the University of Siegen, West Germany under Prof. M. Simon. Beamline hardware, tuning, monitoring and accelerator operations were the responsibility of the Berkeley collaborators. The drift chamber trajectory subsystem was provided by the Siegen group, and LSU had responsibility for the telescope, the mechanical mounting, the logic, the readout system and monitoring during the runs. All groups participated in data-taking. The analysis is done mainly by LSU and Siegen, and the analysis software was developed and run at LSU. Joachim Isbert, a student at the University of Siegen worked both at LSU and at LBL on this project and was awarded his Ph.D. from Siegen at the end of 1991.

For the He-Ne experiments at HISS (P. J. Lindstrom, spokesman), LSU joined the HISS group at LBL and other restarchers from Boston University, The University of New Hampshire, UC-Berkeley, UC-Davis, and UC-Riverside. The HISS group provided overall management of the facility and its configuration for the experiment. LSU was responsible for the upstream monitoring/vectoring hardware and for on-line monitoring software for the scalers. The analysis is centered at LBL with the outside groups sending personnel to work at LBL and/or developing analysis software which is then sent to LBL to be incorporated into the master processing/analysis program. LSU has responsibility for the software for the drift tubes used for the vectoring of the incident particles upstream of the target, for help with the drift chamber analysis, and for work on the overall normalization of the data.

Heavy Beam Fragmentation at HISS required a new collaboration ("TRANSPORT"), which now includes, along with the HISS group, UCBSSL and UC-Riverside, scientists from The University of Minnesota, New Mexico State University, Goddard Space Flight Center, C.E.N. Saclay, France, GSI, Darmstadt, West Germany and Universita di Catania, Italy. The experiment spokesman is T. G. Guzik from LSU. The French group has built liquid hydrogen and liquid helium targets; the Italians are providing a new neutron detection system (MUFNS); the German group will be responsible for the Multiple Sampling Ionization Chamber (MUSIC); Minnesota is in charge of velocity measurements at high energies and LSU developed a beam veto subsystem and was responsible for the trigger logic and for downstream vectoring hardware. Successful data taking runs were completed in Spring, 1990 and Spring, 1991.

Table 1 gives the experimental runs in which our group has taken part, along with some brief comments on each. This year we have added the runs shown in the bottom portion of the table to the "databank." During the past year, we have divided the analysis effort between finishing (1)(3), (5) and (7), performing detailed analysis on (11)-(14), finalizing the results for (11), and doing preliminary work on the new runs (15) - (24). In summary, we now have good datasets for:

- $\quad 160$ at low energies

- Energy dependence of total charge changing cross sections as a function of target mass (28) $\mathrm{Si}$ and beam mass $(\mathrm{Ne}-\mathrm{Ni})$

- A single energy measurement for ${ }^{28} \mathrm{Si}$.

- Several energies for ${ }^{4} \mathrm{He}$ fragmentation studies

- Two-three energy points, with the $\mathrm{LH}_{2}$ target, for the major species ${ }^{32} \mathrm{~S},{ }^{36} \mathrm{Ar}$, and ${ }^{40} \mathrm{Ca}$

- Several energy points for neutron rich beams, ${ }^{22} \mathrm{Ne},{ }^{26} \mathrm{Mg}$ and ${ }^{40} \mathrm{Ar}$

- Single points in the iron peak region for ${ }^{52} \mathrm{Cr},{ }^{56} \mathrm{Fe}$ and ${ }^{58} \mathrm{Ni}$. 
TABLE 1: EXPERIMENTS PERFORMED

Beam Energy MeV/n Eacility Angles Comments

$\begin{array}{llllcc}\text { 1) } & 160 & 225 & \text { B40 } & 6 & \text { limited statistics } \\ \text { 2) } & 160 & 170 & \text { B40 } & 11 & \text { good run } \\ 3) & 160 & 360 & \text { B40 } & 6 & \text { low statistics } \\ 4) & 56 \mathrm{Fe} & 270 & \text { B40 } & 10 & \text { no mass resolution } \\ \text { 5) } & 28 \mathrm{Si} & 1050,550 & \text { B40 } & 1 & \text { limited statistics } \\ & (\Delta \mathrm{Z}=1) & 375,260 & & & 0^{\circ} \text { only, } 5 \text { targets }\end{array}$

6) $\quad 20 \mathrm{Ne} \quad 225 \quad$ B40 $\quad 2 \quad$ unstable beam/low statistics

7) ${ }^{28} \mathrm{Si} \quad 245 \quad$ B40 $\quad 10 \quad$ spill structure/beam problems

8) ${ }^{4} \mathrm{He} \quad 2100 \quad$ HISS $\quad$-- $\quad$ low statistics

9) ${ }^{20} \mathrm{Ne} \quad 2100 \quad$ HISS $\quad--\quad$ good run/DC problems

\begin{tabular}{llcllc}
\hline 10$)$ & ${ }^{36} \mathrm{Ar}$ & 400 & HISS & - & "shakedown" run \\
$11)$ & ${ }^{32} \mathrm{~S}$ & 400,600 & HISS & -- & $\mathrm{LH}_{2}$ target \\
$12)$ & ${ }^{36} \mathrm{Ar}$ & 400 & HISS & -- & $\mathrm{LH}_{2}$ target \\
$13)$ & ${ }^{40} \mathrm{Ca}$ & 400,600 & HISS & -- & $\mathrm{LH}_{2}$ target \\
$14)$ & $56 \mathrm{Fe}$ & 400 & HISS & -- & $\mathrm{LH}_{2}$ target
\end{tabular}

\begin{tabular}{llclll}
\hline 15$)$ & ${ }^{4} \mathrm{He}$ & 400,800 & HISS & -- & $\mathrm{LH}_{2}$ target \\
$16)$ & ${ }^{22} \mathrm{Ne}$ & $400,600,910$ & HISS & -- & $\mathrm{LH}_{2}$ target \\
$17)$ & ${ }^{26} \mathrm{Mg}$ & 400,600 & HISS & -- & $\mathrm{LH}_{2}$ target
\end{tabular}

18) ${ }^{32} \mathrm{~S} \quad 800 \quad$ HISS $\quad--\quad \mathrm{LH}_{2}$ target, low statistics

19) ${ }^{36} \mathrm{Ar} \quad 600,800 \quad$ HISS $\quad--\quad \mathrm{LH}_{2}$ target

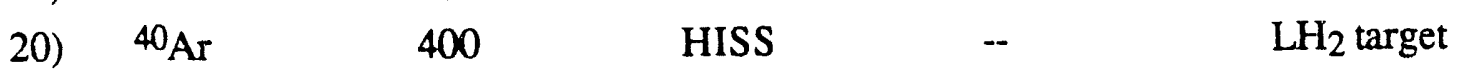

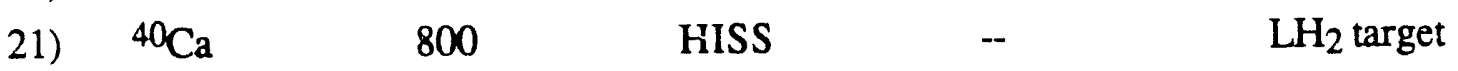

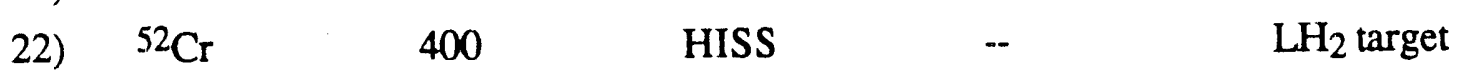

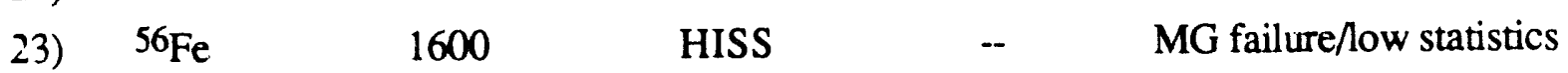

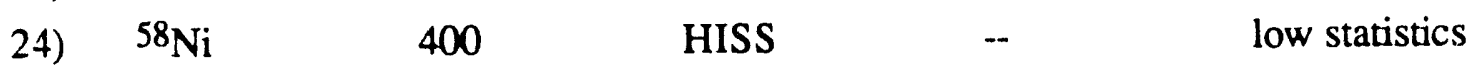

25) ${ }^{56} \mathrm{Fe},{ }^{58} \mathrm{Ni} \quad 600$ Separate Detector System $\quad--\quad$ Thick Targets

-- For (1)-(5) and (7)-(9), $\mathrm{CH}_{2}-\mathrm{C}$ subtraction gives $\mathrm{H}$ cross sections.

-- (6), (10) and probably (23) yield no scientific data.

-- (9) requires extensive software development for the drift chamber. 
The plan for the HISS program had been (a) to extend the current data from runs at $400-800$ $\mathrm{MeV} /$ nucleon to $1.6 \mathrm{GeV} /$ nucleon using the MUSIC detector and a (refurbished) velocity measuring Cherenkov counter (VMD), (b) to obtain an equivalent dataset to Table 1 for a liquid helium target, and (c) to move up the beam mass scale to the $\mathrm{Kr} / \mathrm{Zr}$ and/or Xe/Cs regions. (Theoretically, we should have isotopic resolution for low energy Xe, but actual resolution would depend upon the performance of the detector systems - and this must be investigated experimentally.) This overall program was presented (as a Letter of Intent) to the LBL PAC on 13 December 1991 . The committee view of this program is that it "merits strong support" However, due to the current uncertainty in the future of Bevalac operations, it is unclear whether we will be able to carry out such a long range program. For the next year we are not planning any further runs. We hope that the Bevalac's future will be clarified in the next year, so that experimental datataking can resume -- or an alternative program, e.g. at BNL or SIS, can be developed.

\section{THE APRIL, 1991 HISS RUN}

We were fortunate that circumstances developed which allowed E938H to obtain a long run during April of last year. This resulted from a combination of our having time on the books, the visit and recommended beam line changes of the DOE Tiger Team and a delay in the installation of the EOS TPC. Most of the spring was devoted to this experimental run which used the configuration shown in Figure 2. The drift chamber (DC) was located $\sim 4 \mathrm{~m}$ downstream of the HISS magnet and was followed by the time-of-flight walls (SW and BW). The Italian neutron detector, MUFNS, was aligned with the upstrearn beam path to study undeflected particles.

Upstream vectoring is accomplished by the "Pluto" and "Mickey" counters combined with "Fish" and anti-coincidence detectors UDEW, V1 and V2. The Mickey and Pluto detectors are used to measure the projectile trajectory upstream of the target and the HISS dipole. The Pluto detector is a single sheet ( $1 \mathrm{~mm}$ thick) of scintillator perpendicular to the beam viewed by four phototubes. The Mickey detector is in fact two separate Mickey detectors, each of which is

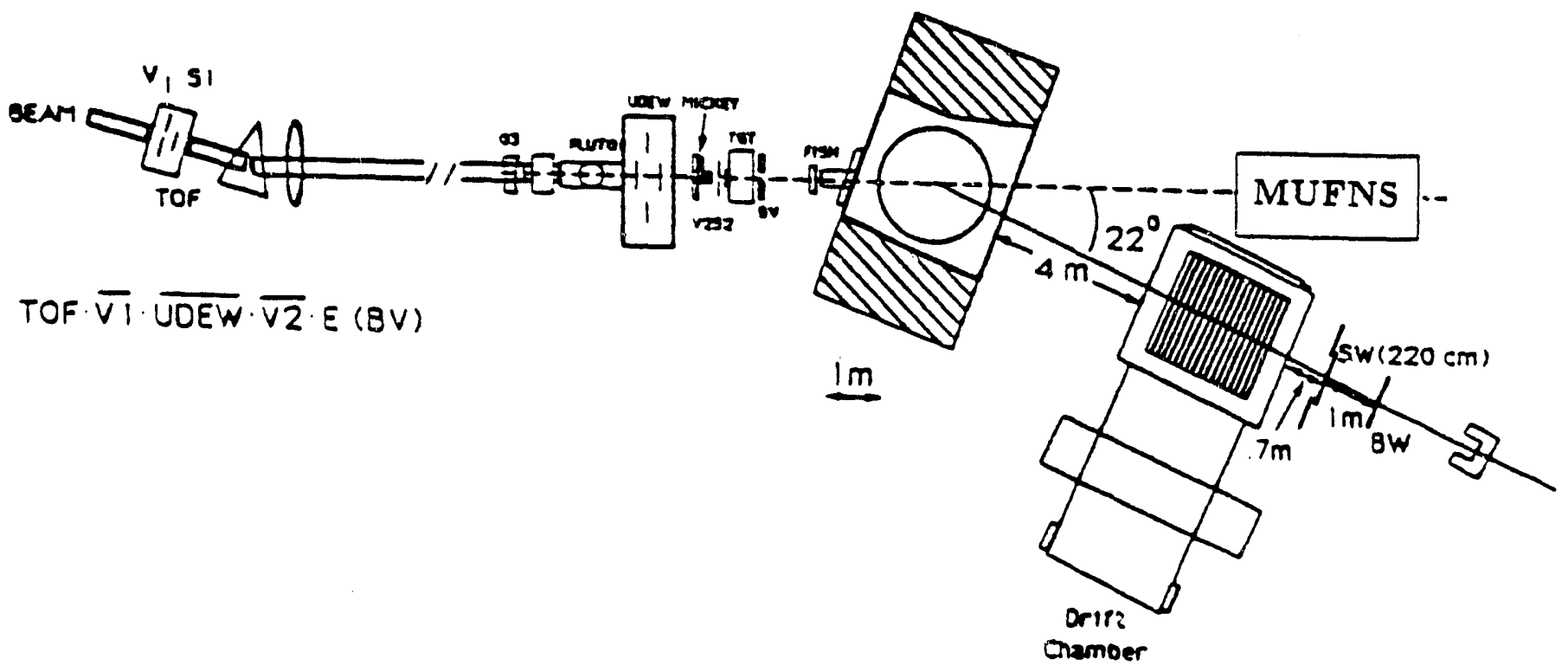

Figure 2. Experimental Arrangement at the HISS Facility. 
composed of a single sheet $(1 \mathrm{~mm})$ of scintillator diagonal to the beam and viewed by two phototubes. The two Mickey detectors are oriented perpendicular to each other and to the beam, one detector providing an $\mathbf{x}$ (horizontal) position measurement, and the other providing a $y$ (vertical) position measurement. The Fish detector is a fiber optic scintillator hodoscope read out by a multi-anode photomultiplier tube. Fish records the position of each fragment as it enters the HISS dipole magnet.

Our French collaborators provided two cryogenic, liquid $\mathrm{H}_{2}$ targets (named "Betty" and "Wilma") and a mass dummy target. In addition, both targets have two condensation chambers of the same diameter but different length. The "Betty" target chambers are of length $3.12 \mathrm{~cm}$, ("thin" target), and $12.1 \mathrm{~cm}$ ("thick" target), while the "Wilma" chambers are $1.87 \mathrm{~cm}$ and $20.09 \mathrm{~cm}$. For the Mar/Apr 1990 run "Betty" was certified to run liquid $\mathrm{H}_{2}$ and only the "thin" region was used. Prior to the 1991 run, the target system was re-built to provide better thermal isolation and the chambers were modified. For $4 / 91$ the liquid hydrogen chamber was $2.4 \mathrm{~cm}$ in length with a $7 \mathrm{~cm}$ diameter. A schematic diagram of the liquid target system is shown in Figure 3. The target was mounted on a moveable platform so that either chamber, or the mass dummy, could be moved easily into the beam line. Alignment between the target and the "nominal" beam line was by survey, but the crucial alignment is between the target chamber and the V1, V2 scintillators which define an acceptable beam particle for the trigger.

Maximizing the number of fragments collected per spill requires identifying and rejecting beam particles that do not interact in the target. This is accomplished by the Beam Veto (BV) subsystem which consists of a $1 \mathrm{~mm}$ thick, 2" diameter solid state detector (SSD) and a $3 \mathrm{~mm}$ thick scintillator viewed by a photomultiplier tube on each cnd. The BV is placed just downstream of the target. The scintillator is employed to provide a fast signal to be used in the trigger.

Discriminators are set so that signals above the threshold are ascribed to beam particles while signals below the threshold are considered fragments. In order to avoid cutting too deeply into $\mathrm{Z}_{\text {beam }}-1$ fragments, the threshold setting only eliminates $\sim 80-90 \%$ of the beam particles. The SSD was too slow to be used in the trigger but was read out for each event. The SSD showed superior charge resolution to the scintillator and was used to both calibrate the scintillator and to provide a measurement of the fragment charge.

As in the previous run, LSU was responsible for the refurbishment, installation and operation of the BV subsystem. In addition, LSU/GSFC nad responsibility for developing and implementing the trigger and readout logic, for calibrating/operating/analyzing the HISS drift chamber, and for set-up of parts of the beam line. LSU pers onnel were at LBL prior to, during and after the actual run period.

The April, 1991 runs involved a step up in energy for many beams to $800 \mathrm{MeV} /$ nucleon. This required re-tuning the BV thresholds and produced some concern about the resolution. Figure 4 shows on-line results for ${ }^{36} \mathrm{Ar}$ at $800 \mathrm{MeV} /$ nucleon. The top figure plots the SSD in the BV system versus the sum of the two tubes on the scintillator. Good charge resolution is evident in this plot and is displayed in the SSD histogran in the bottom figure, where elements down to $\sim$ Oxygen can be clearly distinguished. The $\mathrm{Z}_{b}-1$ element, Chlorine, is clearly affected by the BV thresholds and will have to be corrected for the efficiency. Note that this is raw data which will be improved when background is removed and the calibrations are fine-tuned.

For the spring 90 data we developed a relatively simple procedure to obtain "pseudo-rigidity" or "pseudo-mass" from the data (see Technical Progress Report DOE/ER/40147-9). this was implemented on-line for the 91 runs, and Figure 5 shows an example of the mass resolution observed on-line for ${ }^{36} \mathrm{Ar}$ at $800 \mathrm{MeV} /$ nucleon. Isotope peaks are resolved down to silicon for which the resolution is becoming marginal. This indicates that (a) a strict clean-up of the data will be needed, and (b) a full rigidity analysis using the detailed drift chamber calibration will be required to obtain the best resolution for the lighter fragments. 


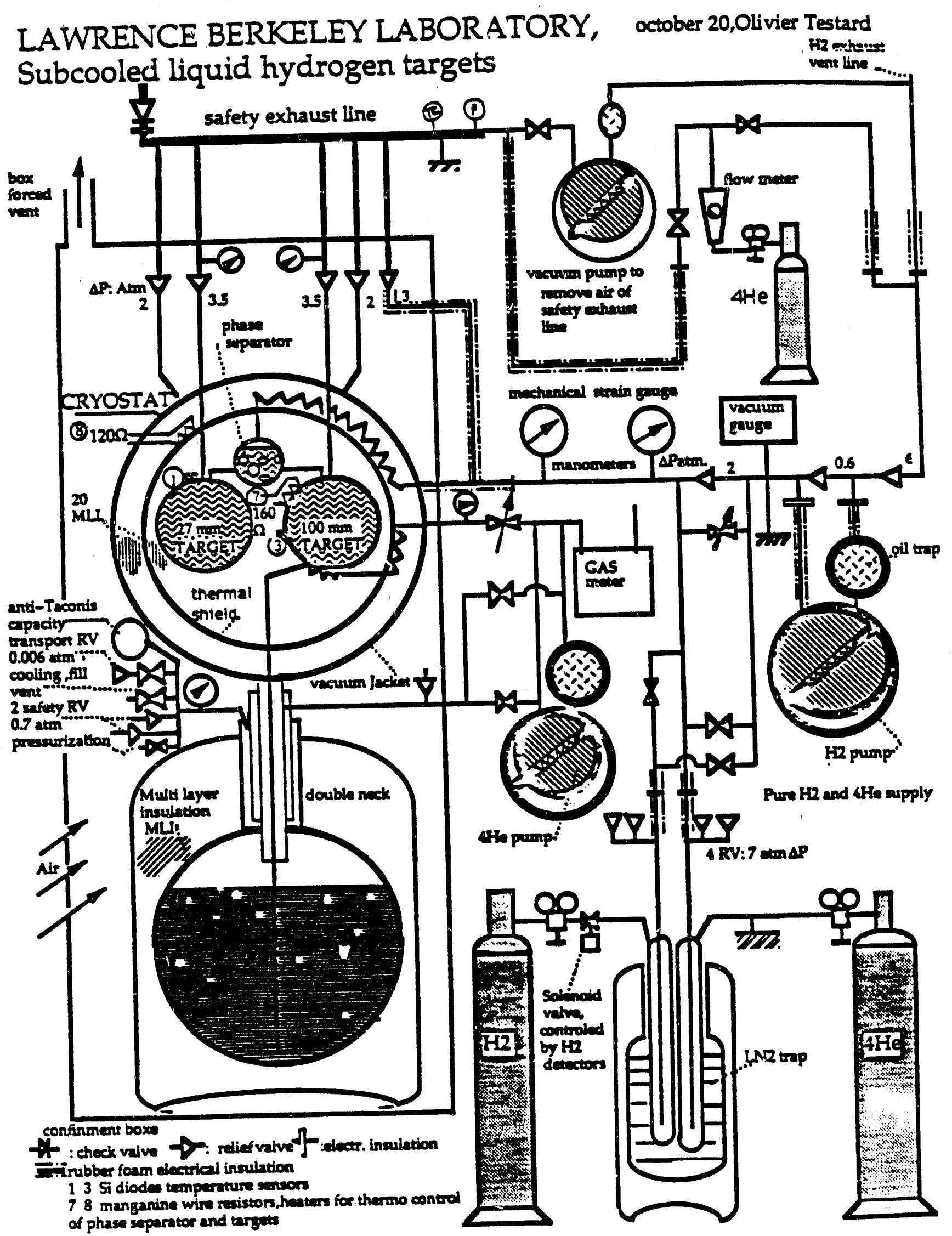

Figure 3. The Liquid Hydrogen Target System. 
Online Data from April, 91 Runs
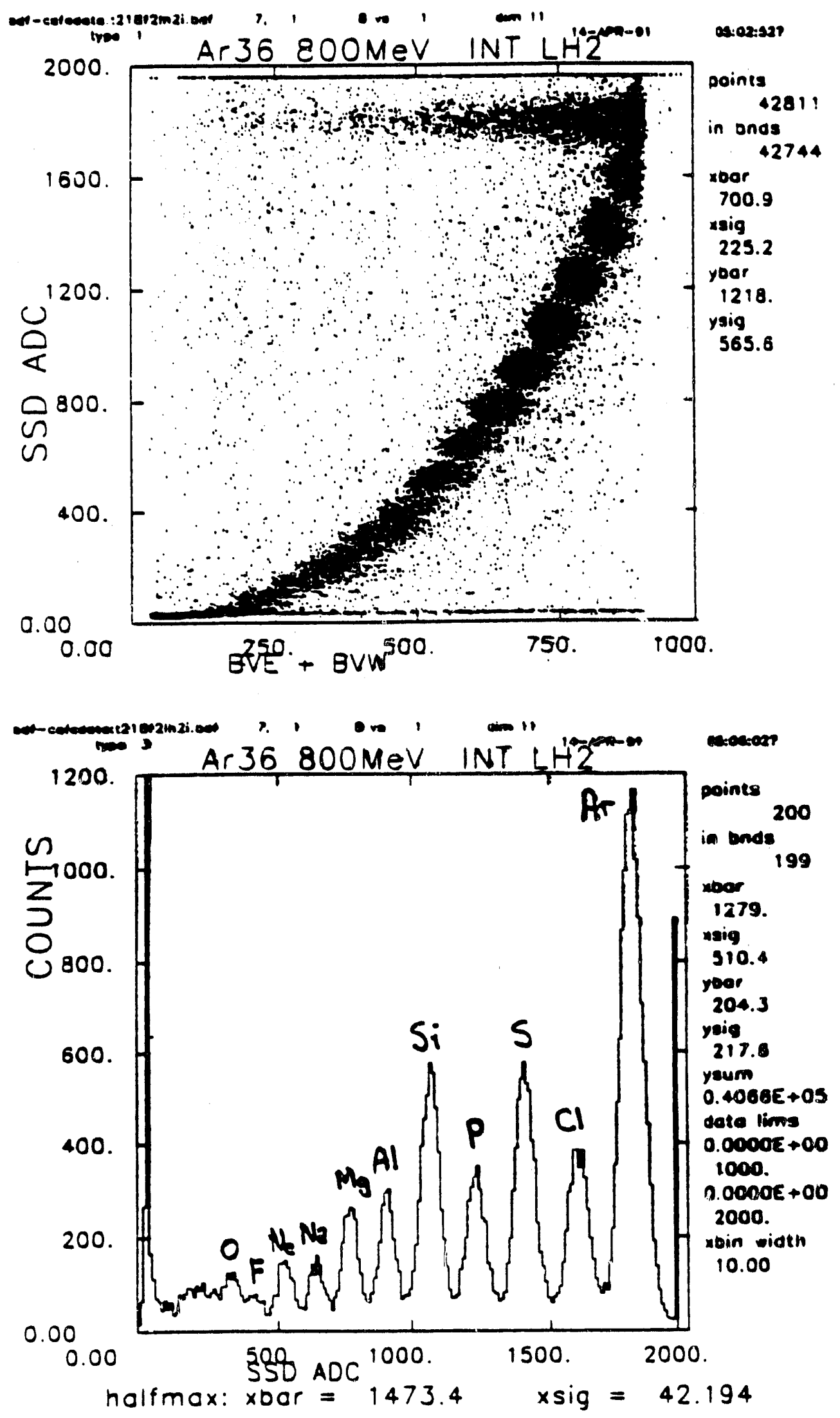

Figure 4. BV subsystem performance for ${ }^{36} \mathrm{Ar}$ at $800 \mathrm{MeV} /$ nucleon. 


\section{Fragment Mass Histograms (Data taken online during q/gl runs)}
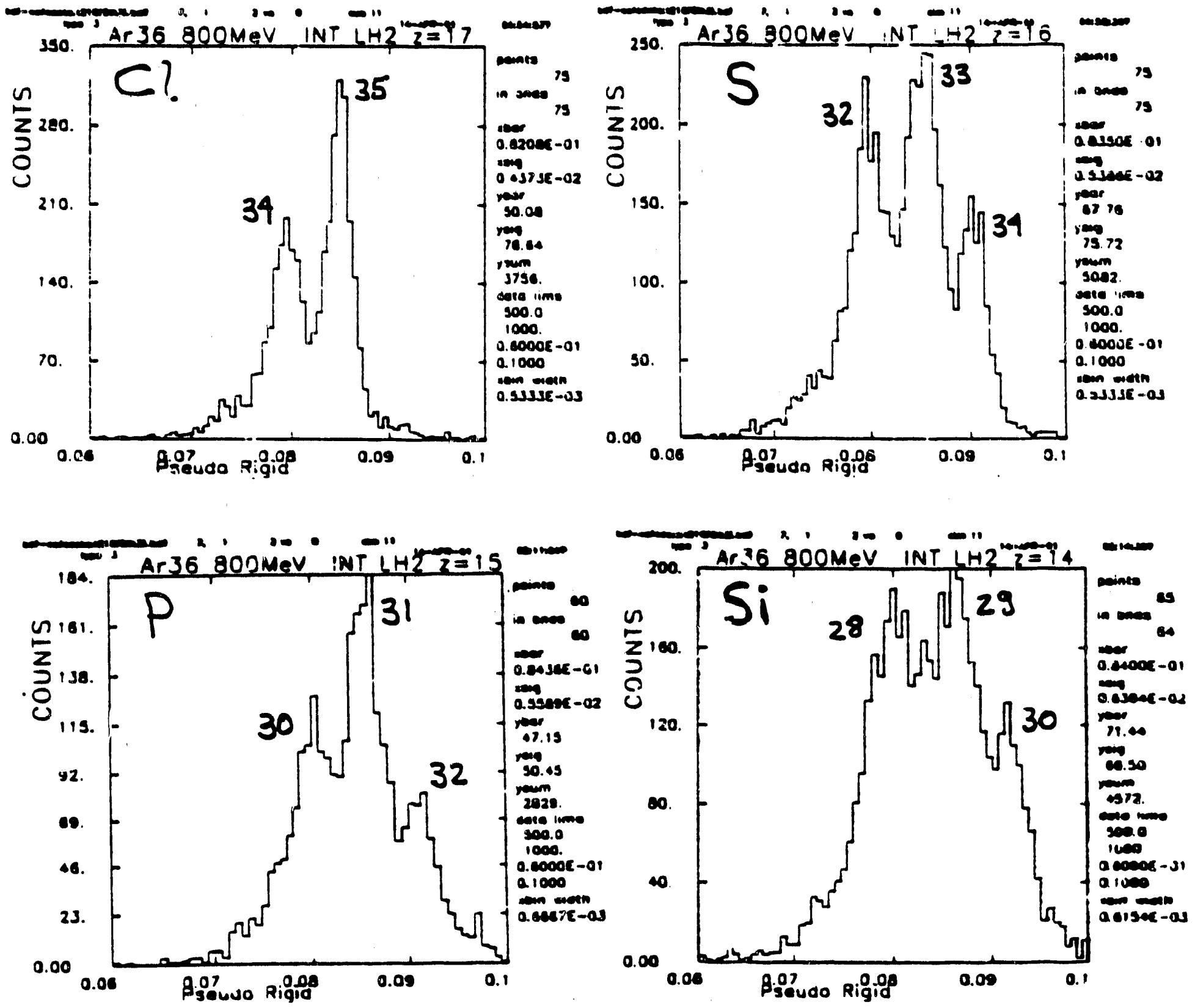

Figure 5. Pseudo-rigidity distributions obtained for ${ }^{36} \mathrm{Ar}$ at $800 \mathrm{MeV} /$ nucleon. 


\section{ANALYSIS PROGRESS}

\section{A. HISS Runs}

\section{Data Reduction, Processing and Analysis}

With the addition of $\sim 15$ new datasets this past year, it was necessary to establish more formal procedures for data handling. The goal was to free senior personnel for the detailed analysis and allow the junior people to proceed with reduction and processing with a minimum of supervision. To this end, we have adopted a three level scheme as follows:

Level 3: Phase 1 (Preliminary) Analysis -- This begins with the raw data tapes produced on-line and involves, first, copying and checking. This is done at LBL following the data-taking runs and insures a readable dataset. At LSU the $8 \mathrm{~mm}$ tapes are read, structural defects are corrected, event statistics are generated, headeis added as needed, irregularities are noted and then 6250 BPI tapes and $8 \mathrm{~mm}$ tapes are produced. Muitiple $8 \mathrm{~mm}$ copies are made and distributed to the collaborating institutions. This completes the archiving/distribution function. Note that, except for structural defects (e.g. missing EOF marks), these tapes contain raw data. This function has been completed for all of the HISS datasets.

Preliminary analysis is also performed at this level. Charge calibrations are determined for the BV subsystem and for the ToF walls, preliminary trajectory results are obtained and beam geometry monitoring is performed. The results of these analyses are subroutines that read the raw data and produce the first order physics parameters.

Level 2: Phase 2 (Detailed) Analysis -- This is the longest phase in the analysis process and involves the senior personnel working together and with the undergraduate junior analysts. Here the calibration routines for each subsystem are finalized, the upstream vectoring and alignments are used to determine the acceptance, the $x-y$ position of each event is determined, the ToF is evaluated and the drift chamber irajectory is evaluated. Background is rejected by requiring consistent charge values between the $\mathrm{BV}$ and ToF and the efficiencies and overall normalization are determined. At this stage the subroutines developed in Level 3 and Level 2 work may be combined into a processing program which is run on the full dataset. This produces a new data volume containing the good events and all of their associated physics parameters. Such a "processed" data set is then used for further refinements and physics analysis. During the past year about $1 / 3$ of the datasets have been moved into this Level 2 ana'ysis.

Level 1: Publication Preparation -- At this level a specific physics goal has been identified and the analysis is directed tow'ards producing publishable results. This requires the details of the normalization to be worked out, and an uncertainty analysis performed. Sometimes this may reveal problems that require dropping back to Level 2 for some period of time. Documenting the final analysis procedures and results is also a part of the Level 1 work. The "output" from Level 1 will be manuscripts to be submitted for publication. Currently we have the elemental analysis and the isotopic cross sections for ${ }^{32} \mathrm{~S}$ in Level 1 analysis, and the preliminary results are described below.

\section{Elemental Analysis:}

The first parameter which must be determined for each event is the charge of the particle emerging from the target. This is done initially by the BV subsystem and confirmed by the ToF wall anaiysis. The BV records events very close to the target, so that secondary interactions are minimized (basically only the thin target flask windows contribute to the background). What is required is to analyze the SSD and the BV scintillator, as was shown in Figure 4, and to correct for background events, beam spill-over, and charge dependent efficiencies from the threshold cuts. 
This then produces a clean charge spectrum emerging from the target. For each beam-energy run, data were taken for both target full and target dummy configurations which allows the secondary contribution from the windows to be determined.

It should be noted here that, for each run, data were taken both with the BV subsystem and with the BV trigger disabled. The latter runs record all particles with uniform efficiency for both beam and fragments. These non-BV runs are used to establish the charge dependent efficiencies as well as to calibrate the overall normruization of beam to fragments.

Since much of this type of analysis is performed at Level 3, it was decided that pushing such a study through Levels 2 and 1 would be valuable. This not only checks the normalization and efficiencies of the pre-magnet system but also provides an indication of any problems in the data or its interpretation, which will be important to know before the detailed isotopic analysis is undertaken. In avidition, the results are scientifically important since they can be compazed to previous data and to models that attempt to predict the cross section values.

Starting with cleaned histograms $u$ ch as the lower part of Figure 4 each element peak was fit to a Gaussian distribution. The analysis was extended at least to $\mathrm{Z}_{b} / 2$ and lower in the cases for which peaks were identifiable. in addition, a "count" of the total number of incident beam particles was obtained. Using the ratio of the number of events, the total charge changing cross section can be determined. Figure 6 (top) displays sone of the preliminary results compared to two predictive formulae and to previous measurements. Where comparison is possible, our results are in good agreement with previous work. The $\mathrm{A} / \mathrm{Z}=2$ species also show agreement with theory for energies $\geq 600 \mathrm{MeV} /$ nucleon. What is interesting is the disagreement of the neutron rich species $22 \mathrm{Ne}$ and $26 \mathrm{Mg}$ with theory at high energies and the low values found consistently for the $400 \mathrm{MeV} /$ nucleon points.

The lower part of Figure 6 shows a summary of all of the data that have been analyzed, plotted versus $A_{\text {beam. }}^{2 / 3}$. As is well know, $A^{2 / 3}$ organizes the data, and the higher energy results appear to follow curves with about the same slope. The $400 \mathrm{MeV} /$ nucleon data, however, are consistently low and follow a line with a different slope. We have shown previously (with ${ }^{28} \mathrm{Si}$ ) that there is an energy dependence to total cross section, both p-A and A-A, but the degree of the dependence indicated by the $400 \mathrm{MeV} /$ nucleon data is surprising. Thus, we are left with a question, "Is it physics or daia analysis?"

Turning next to the individual element cross sections measured for beams of ${ }^{22} \mathrm{Ne},{ }^{32} \mathrm{~S}$, $36,40 \mathrm{Ar},{ }^{40} \mathrm{Ca}$ and ${ }^{58} \mathrm{Ni}$, Figure 7 shows a global comparison between our measured values and those predicted by the semi-empirical formulae of Silberberg and Tsao and the Parametric equations of Webber et al. Overall the distribution of measured versus predicted values is tighter for the parametric equations, but significant deviations, especially for elements with large cross sections are observed in both cases.

The fragment yields for the various beams and energies are displayed in Figure 8 where the top panel compares a neutron rich beam ${ }^{22} \mathrm{Ne}$ to an $\mathrm{A} / \mathrm{Z}=2$ beam ${ }^{32} \mathrm{~S}$. The lower panel cumpares different isotopes of Argon, the $A=40$ isobar and the heaviest beams. Pronounced even-odd effects are observed for some species, but, surprisingly, not for others $\left({ }^{22} \mathrm{Ne},{ }^{40} \mathrm{Ar},{ }^{52} \mathrm{Cr}\right)$. Note also that the $400 \mathrm{MeV} /$ nucleon points for ${ }^{22} \mathrm{Ne}$ and ${ }^{32} \mathrm{~S}$ diverge from the higher energy results, particularly at small fragment charge.

Thus, there appears to be potentially interesting physics in the element analysis. Confirming these preliminary results as physics effects has been and will continue to be one of our chief Level $i$ andilysis effurts. 

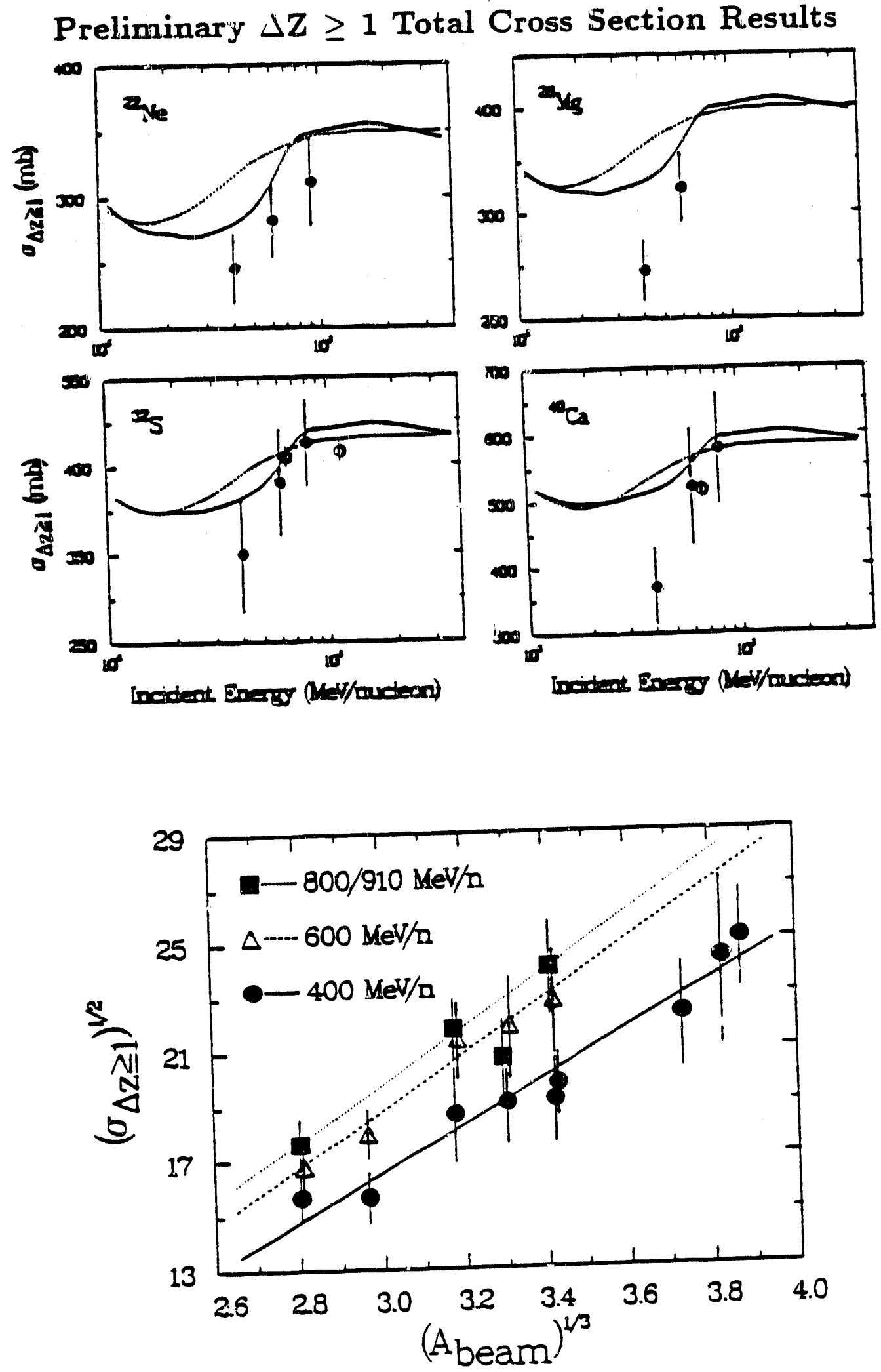

Figure 6. Preliminary total charge changing cross sections as a function of energy (top) and the cross sections versus $A^{2 / 3}$ (bottom). 

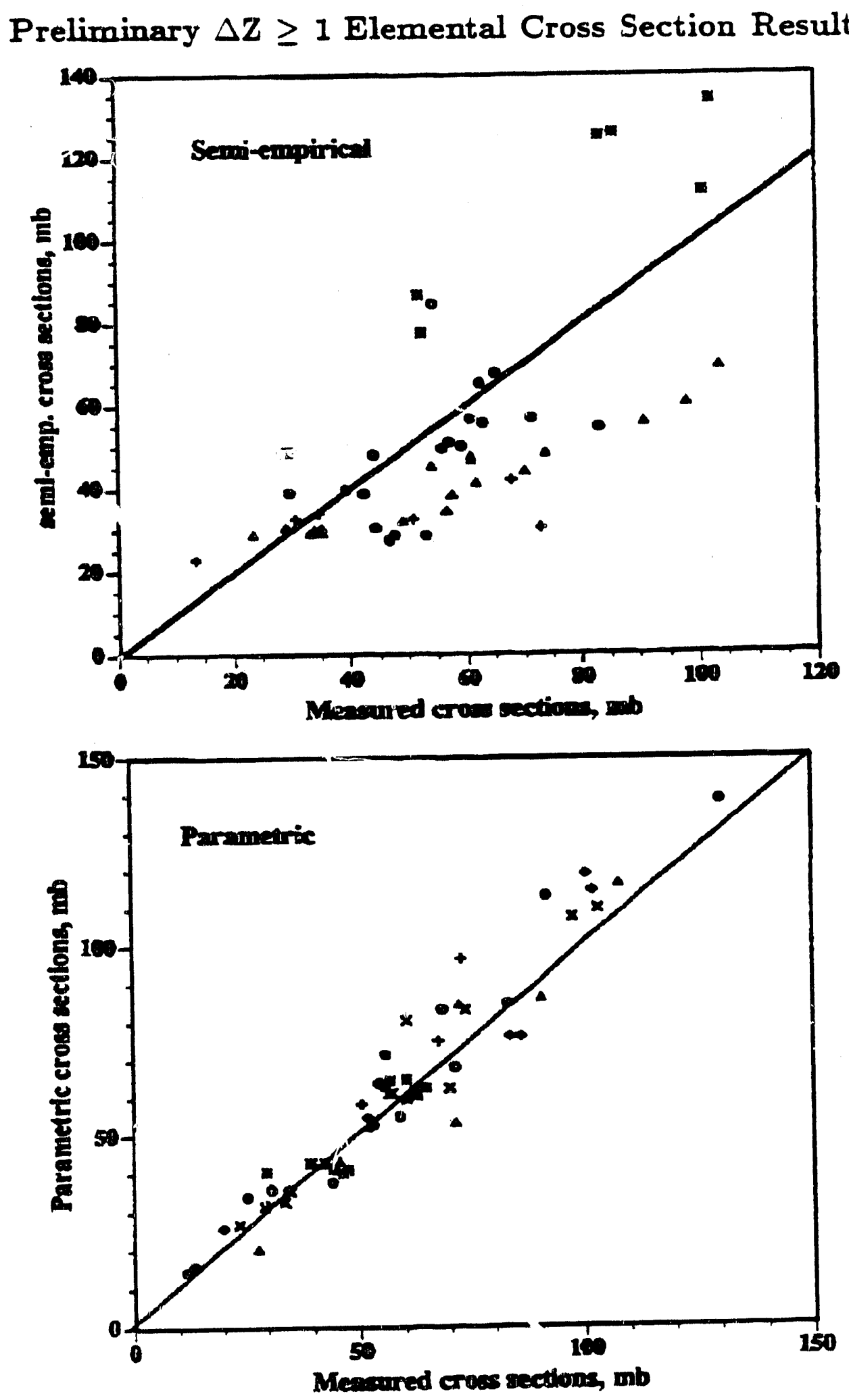

Figure 7. Comparison of measured elemental cross sections to predications from the semiempirical formulae (top) and the Parametric equations (bottom). 
Preliminary $\Delta Z \geq 1$ Elemental Cross Section Results
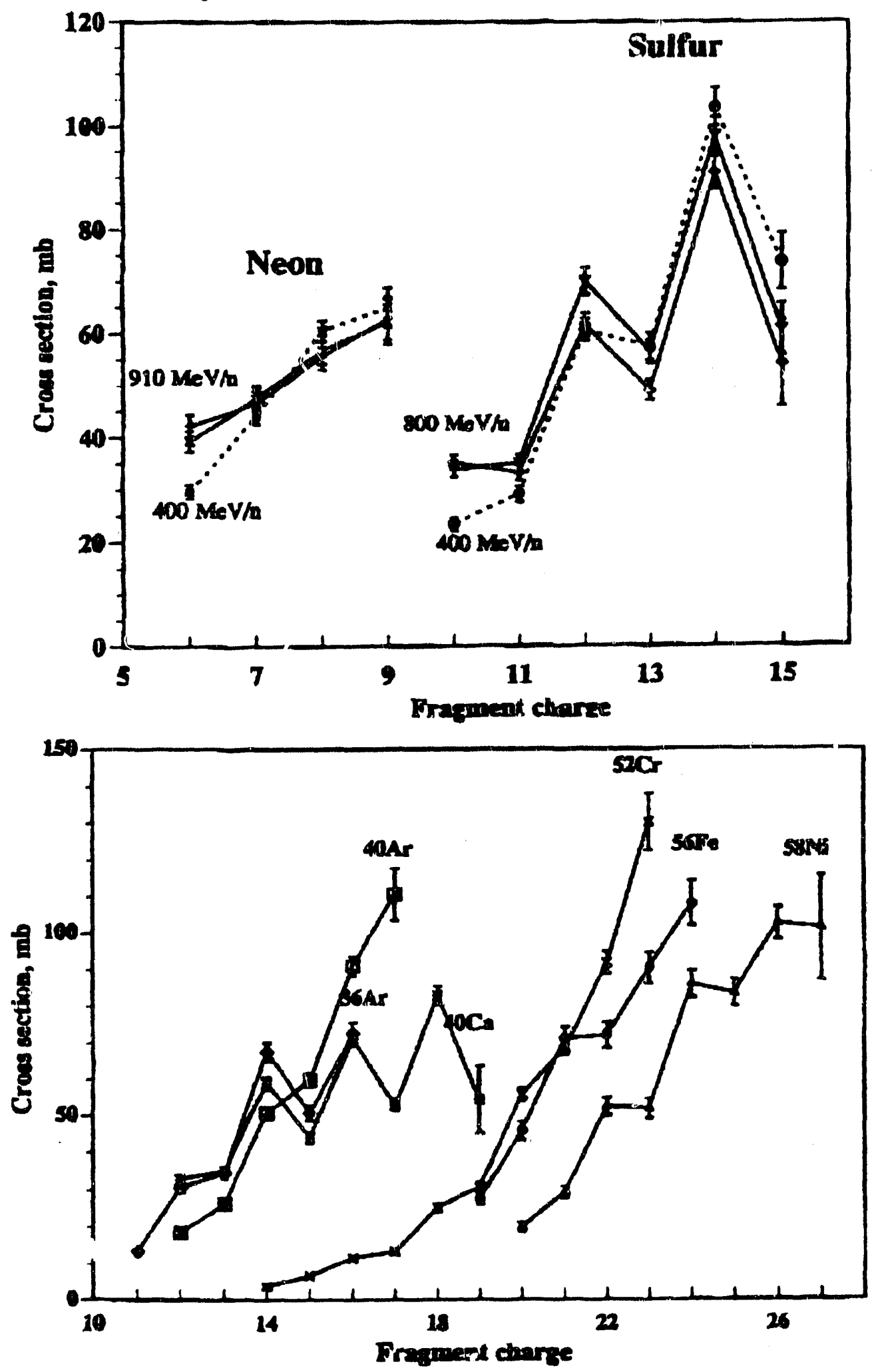

Figure 8. Element yield functions for ${ }^{22} \mathrm{Ne}$ and ${ }^{32} \mathrm{~S}$ (top) at a variety of energies and for other species at $400 \mathrm{MeV} /$ nucleon (bottom). 


\section{Beam Distributions and Target Effects:}

The low values determined at $400 \mathrm{MeV} /$ nucleon (c.f. Figure 6) have prompted us to reexamine the normalization and the efficiencies. One of the critical parameters in the absolute cross section (as well as in the uncertainty) is the thickness of the liquid hydrogen column traversed by the beam. The hydrogen flask is nominally a cylinder with thin titanium windows on either end. The target is operated at $\sim 2$ atmospheres which causes the end caps to bow outward (depending upon the exact pressure), as illustrated in Figure 9. While the monitoring equipment held the temperature and pressure of the liquid hydrogen constant, so that the density was $0.07 \mathrm{~g} / \mathrm{cc} \pm 1 \%$ during the run, the exact linear thickness experienced by the beam depends upon its location on the target cylinder face and upon the beam size and distribution. (Maximum center to edge variation is as large as $25 \%$ !) Since the beam parameters vary from one energy tune to the next, it is possible to introduce an energy dependent systematic effect into the data through a varying position on the target ( $\sigma$ proportional to $t^{-1}$ ).

Beam definition is determined, near the target, by the anti-coincidence scintillators V2 and V4 as illustrated in Figure 10. Using the surveyed positions of these detectors, we predict the alignment shown in Figure 11 for the 4/90 runs. The beam spot determined by V2 is definitely offset from the center of the target. What remains to be studied is the beam tune, particularly the divergence between V2 and V4 and the distribution of the beam over the V2 "hole."

A similar analysis must be performed for the April 91 configuration, shown in detail in Figure 12. The raw survey results are shown in Figure 13 and indicate some significant misalignments, although in this case it appears to be more in the E-W rather than the U-D direction.

Analyzing the beam profile requires use of the Mickey and Pluto detector systems (c.f. Figure 2). These provide, when fully calibrated, an E-W and U-D position for each particle. This will give both the beam profile and the average divergence for each run. The difficulty is the absolute calibration of these systems, which is currently a major area of investigation.

A related problem is the acceptance of the V4 and BV subsystems. At the lower energies, fragments may be emitted at relatively large angles to the incident particle direction. In such cases, it may be possible to miss the V4 hole (c.f. Figure 11 for a fragment emitted upward) or the BV detectors. This would lower the absolute fragment yields, becoming worse for the lower- $Z$ fragments. (We have observed such large angle emission during the B40 experiments at lower energies.) Here the FISH detector, which records the position of each fragment downstream of the target, could be the critical component and work is being undertaken to understand the mapping and the calibration of this detector system.

As expected, the elemental analysis has pointed to a number of areas needing further study. In this sense, it has served its major purpose, since these effects must be understood before full isotopic analysis is undertaken. In addition, the studies currently in progress will lend credence to the elemental results and will verify (or refute) the effects that were noted in the preliminary results.

\section{Preliminary Isotopic Results:}

Simultaneously with the Level 3 work and the Level 2 studies of the elemental cross sections, we have continued to pursue the full isotopic analysis using the $4 / 90$ run of $32 \mathrm{~S}$ at 400 $\mathrm{MeV} /$ nucleon as the dataset for analysis. The effects being learned from the elemental analysis are included as they are derived, and the on-going k'ork is designed to produce the techniques and software that will be needed for the isotopic analysis of the other datasets. 


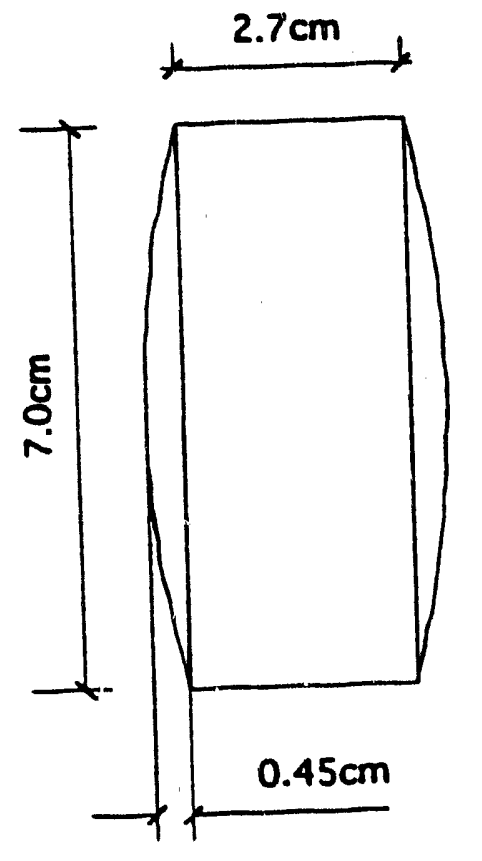

Figure 9: $\mathrm{LH}_{2}$ Flask
V2
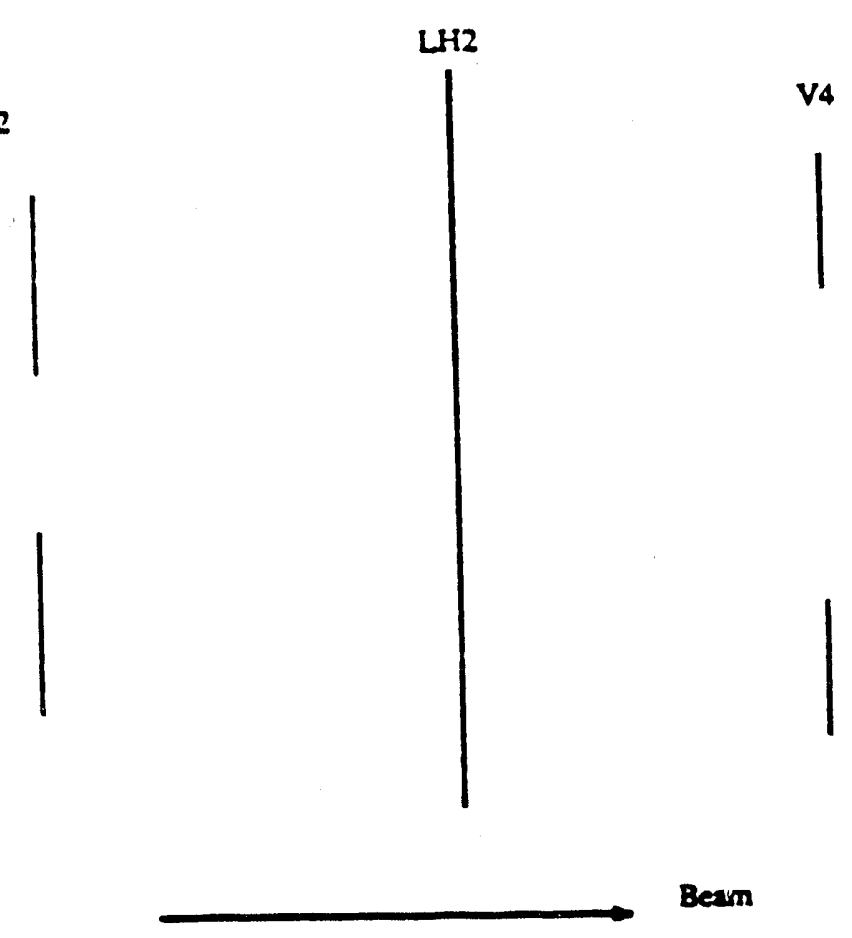

Figure 10. Anti-coincidence Scintillators.

\section{Front View}
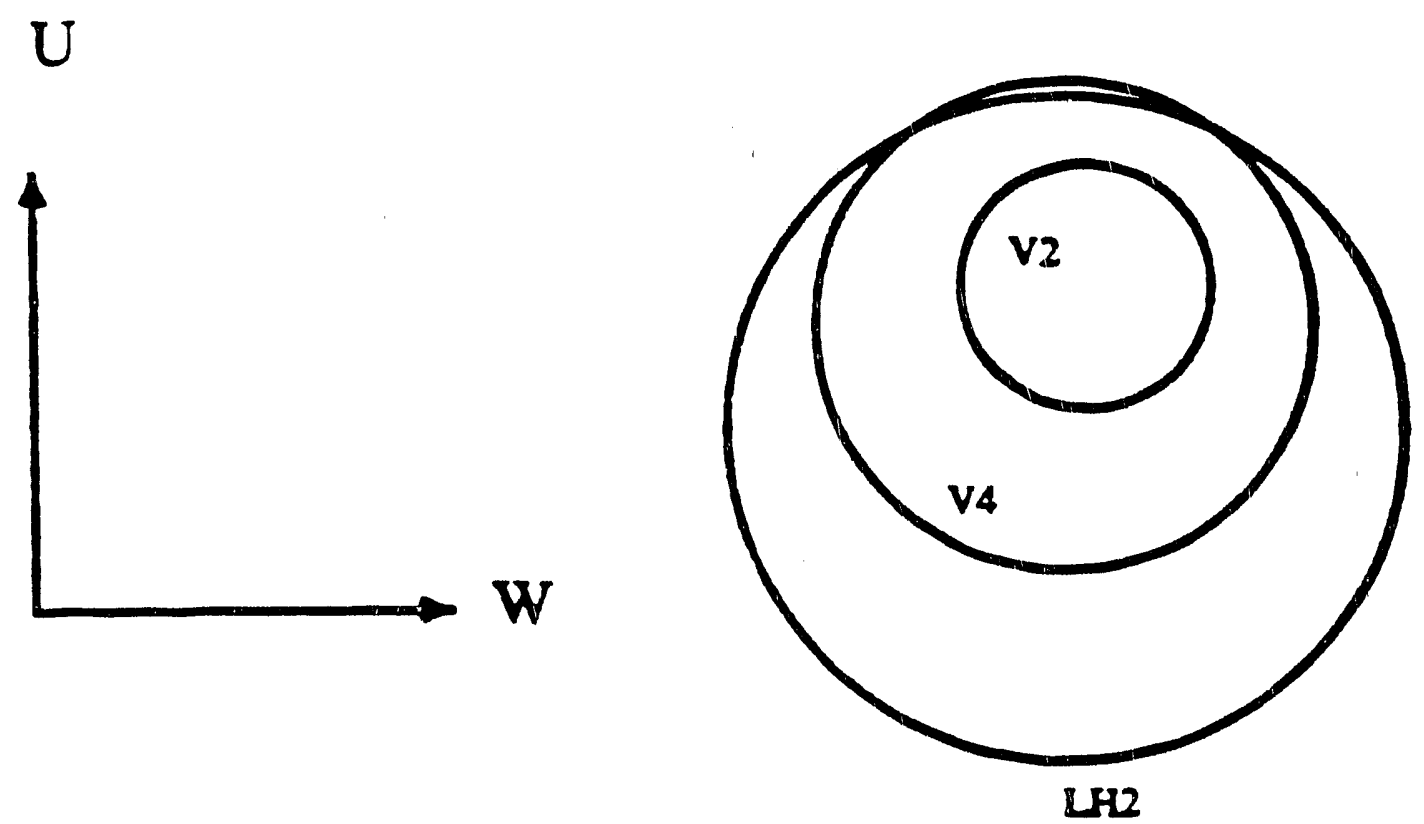

Figure 11. Preliminary alignment for April, 90 runs. 


\section{Beam Definition System}

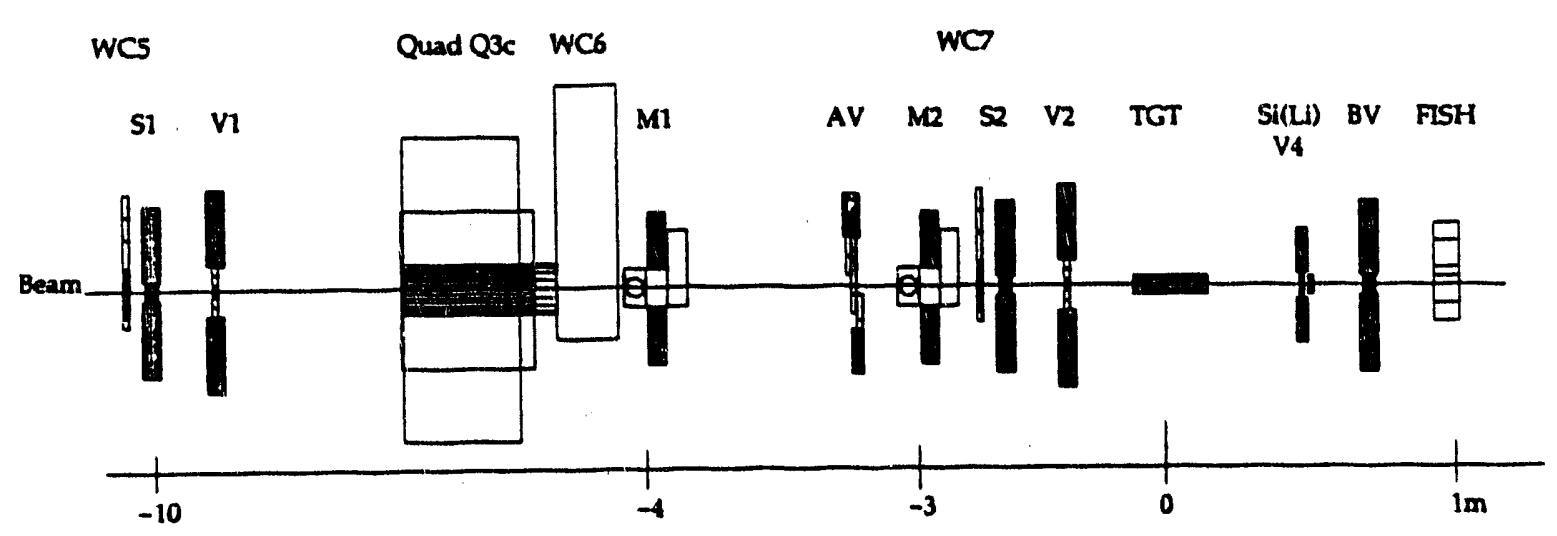

Figure 12: Full beam definition system.

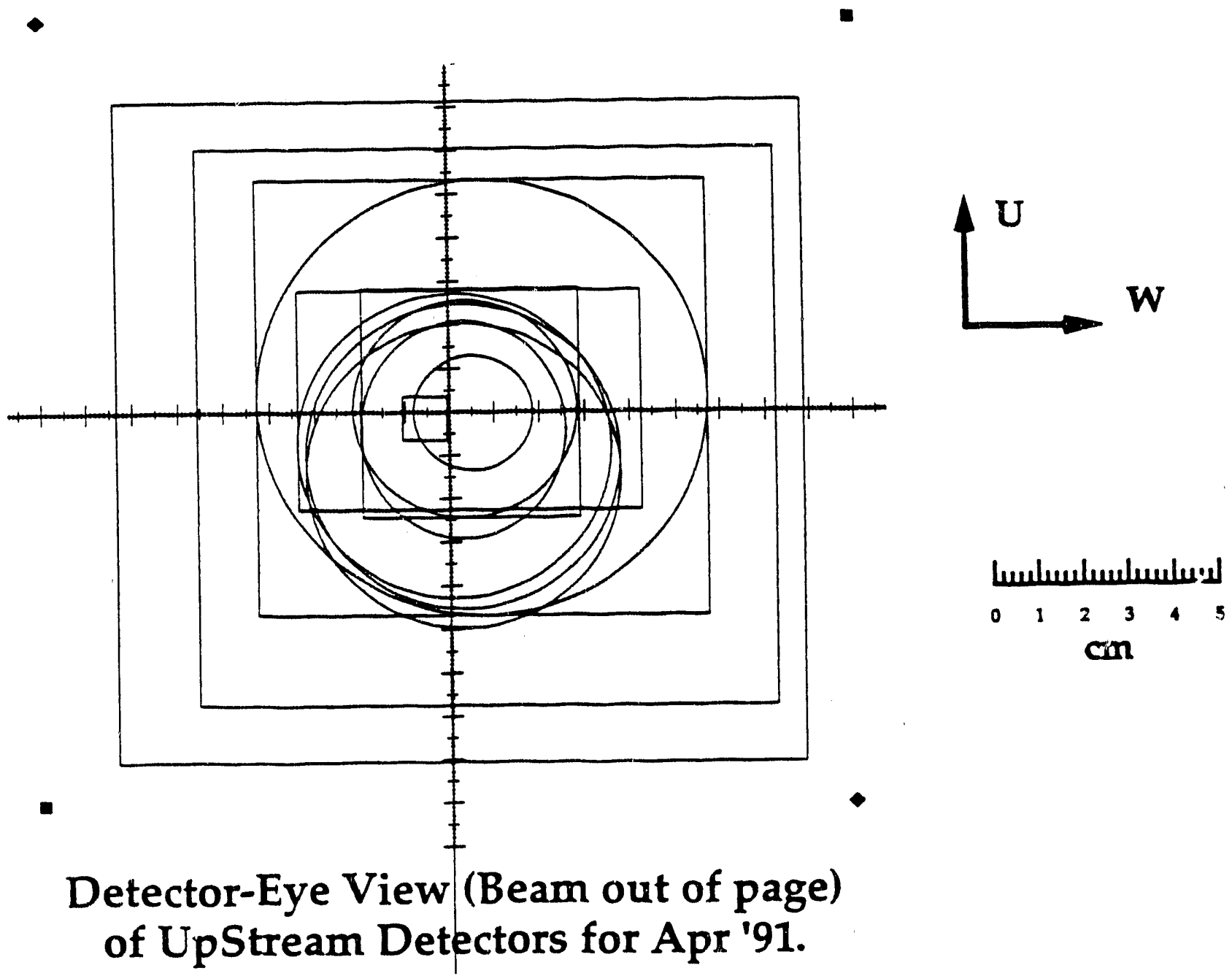

Figure 13: Preliminary alignments for April, 91 runs. 
The current state of the mass resolution is illustrated in Figure 14 which shows the isotope peaks for fragments from $Z=15$ through $Z=7$. The mass resolution here is $\sim 0.2 \mathrm{amu}$. The analysis uses the ToF wall for velocity measurements, background rejection and coarse positioning and uses the DC for pseudo-rigidity determination (more information is available from the DC but at an analysis cost of fine-tuning the time-to-space functions for each DC plane). The improvement in Figure 14 compared to the results of last year, or compared to the on-line results shown in Figure 5 , is dramatic.

Using the most current normalization and hydrogen target results, these histograms have been converted to cross sections in order to compare the mass yields to the predictive formulae in current use. These comparisons are shown in Figure 15 where the top plot compares the data (dashed lines) to the semi-empirical formulae of Silberberg and Tsao (solid lines) and the lower plot gives a similar comparison to the parametric equations of Webber et al. The top plot shows larger experimental yields for the high-A fragments relative to the formulae, while the bottom plot shows deviations mainly for the neutron-rich and neutron-poor isotopes, in the cases for which comparisons are possible. Overall our preliminary data agree better, for the major isotopes, with the Webber et al. results than with the Silberberg and Tsao predictions. However, there are still deviations that are outside the experimental uncertainties!

The $400 \mathrm{MeV} /$ nucleon ${ }^{32} \mathrm{~S}$ analysis appears to be close to completion. Including final efficiencies, target thickness and beam effects should be possible in the next few months. This will yield a final dataset that is ready for publication.

\section{B. B40 Data}

The B40 data has been under analysis for several years principally at LSU ', nd The University of Siegen, Germany. These experiments utilize the spectrometer var.uum tank in the B40 area to spread the fragments based upon their transverse momenta. The detector system, a silicon solid state detector telescope that brings the particles to rest in the stack, is mounted on a cart that can be moved along the rail to trace out the angular distribution of the fragments. Cross sections are obtained by integrating under the angular distributions and subtrayting the target out background results. Targets of $\mathrm{C}$ and $\mathrm{CH}_{2}$ (among others) are used, allowing, $\mathrm{H}$ cross sections to be ottained by $\mathrm{CH}_{2}-\mathrm{C}$ subtraction.

The silicon solid state detectors provide excellent mass resolution by the $\Delta \mathrm{E}-\mathrm{E}$ technique, as illustrated in Figure 16 for fragments of 160 . Particle trajectories are determined by positionsensing solid state detectors $\left({ }^{16} \mathrm{O}\right)$ or a multi-plane drift chamber $\left({ }^{28} \mathrm{Si}\right)$ located ahead of the solid state detector stack. The drift chamber development was spearheaded by our Siegen collaborators, and Dr. Joachim Isbert was awarded the Ph.D. degree this past year for his work on this experiment.

Thesis: $\quad$ "Experimentelle Bestimmung der Wirkungsquerschnitte von Silizium an Wasserstoff bei einer Energie von $245 \mathrm{MeV} /$ nukleon," UniversitätGersamthochschule Siegen, November, 1991 (unpublished).

Cross sections have been determined on both hydrogen and carbon targets for fragments from $\mathrm{Mg}$ to $\mathrm{B}$. Uncertainties for the major cross sections are $\leq 10 \%$. Figure 17 compares these new results to the predictions of the Silberberg and Tsao semi-empirical formulae for each of the isotopes. In this plot a $20 \%$ error has been assigned to the ratios of the cross sections (measured/calculated). The striking result is that, overall, the calculations underpredict the cross sections, even for the major isotopes of a given element. The average ratio is about 1.5 but there is apparent structure, as a function both of fragment charge and of mass within each element. 

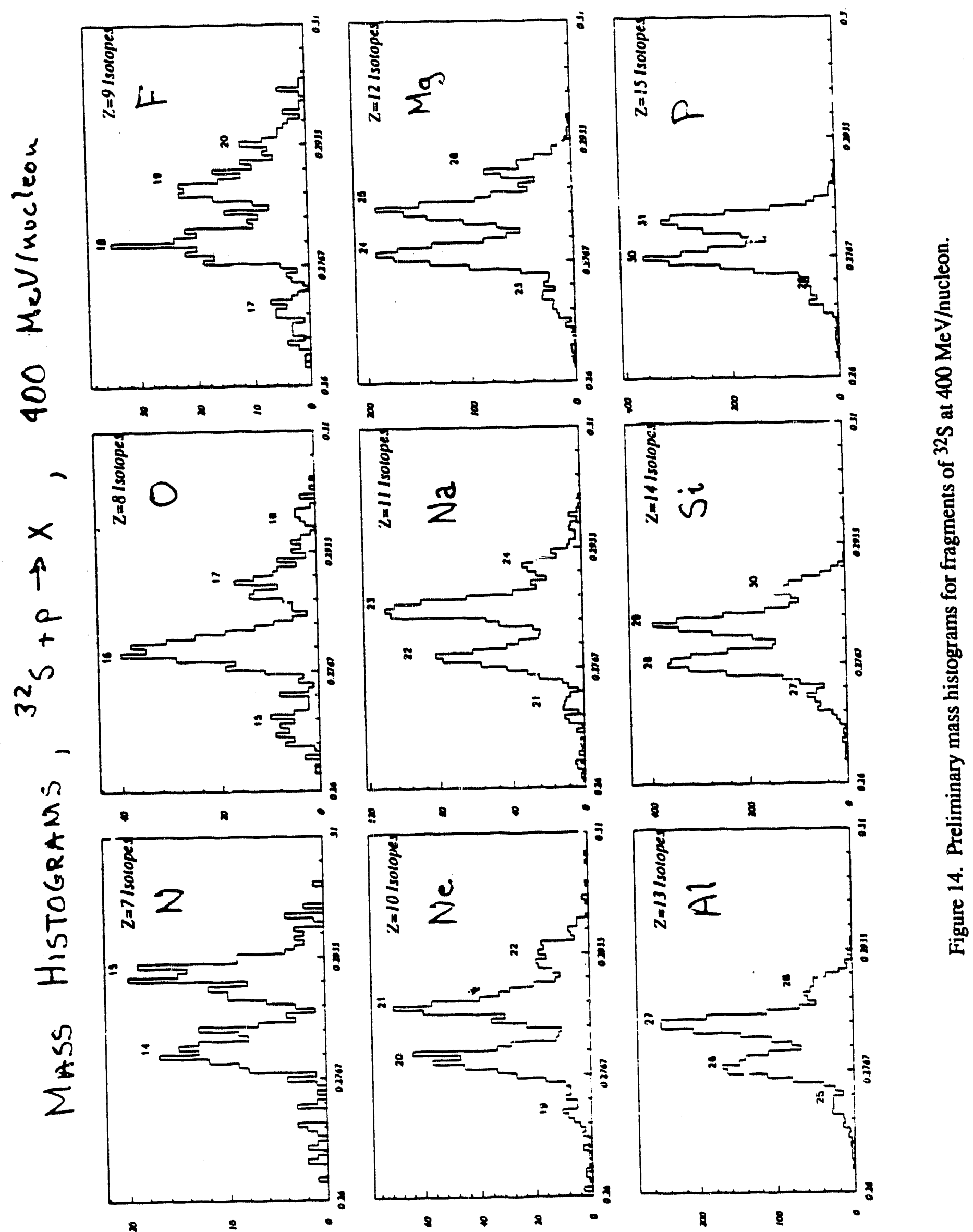
Freliminary $\Delta M \geq 1$ Isotopic Cross Section Results
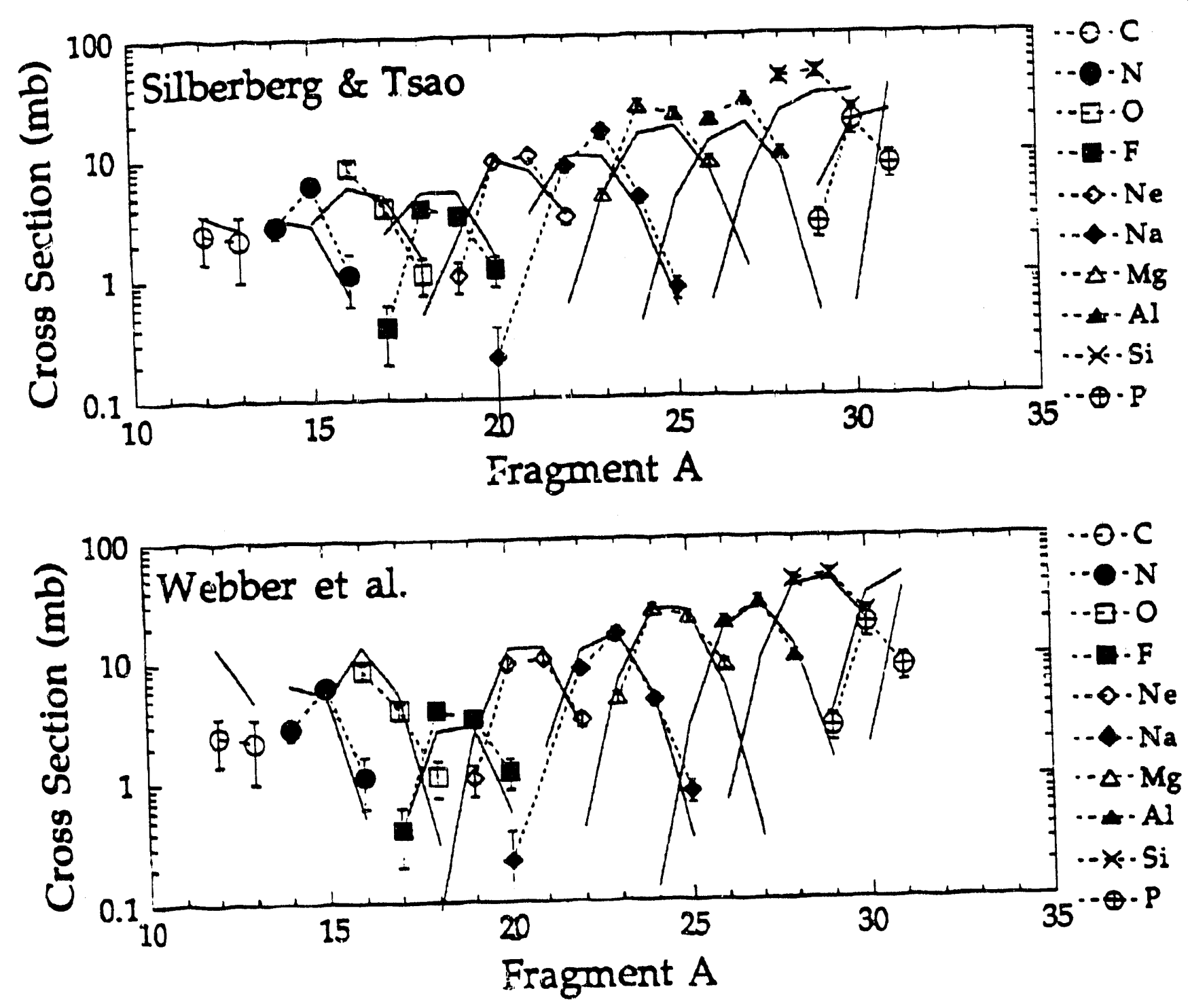

Figure 15. Comparison of experimental mass yields to predictions for the fragments of ${ }^{32} \mathrm{~S}$ at $400 \mathrm{MeV} /$ nucleon. 

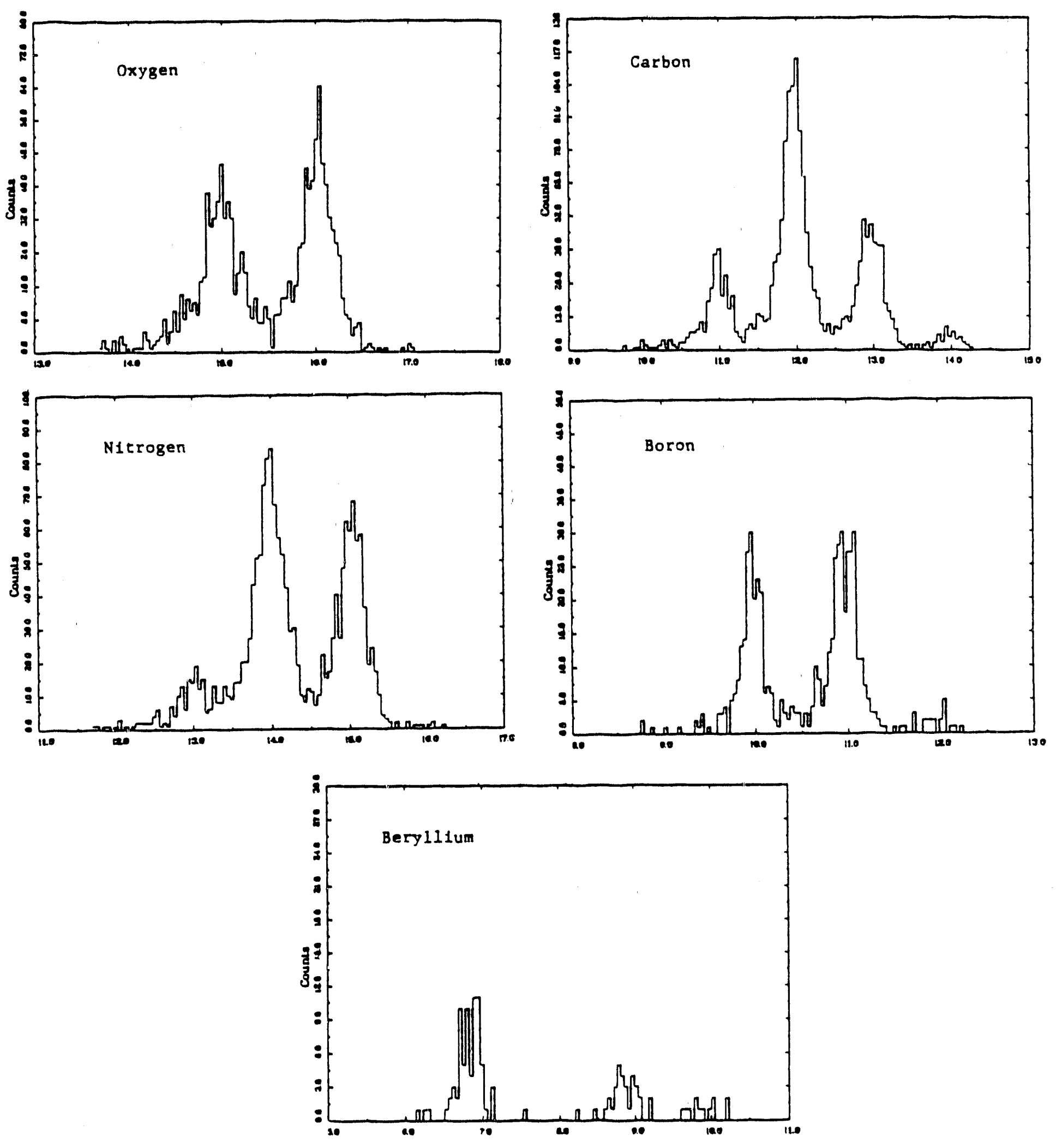

Figure 16. Mass histograms for fragments of 160 at $160 \mathrm{MeV} /$ nucleon. 


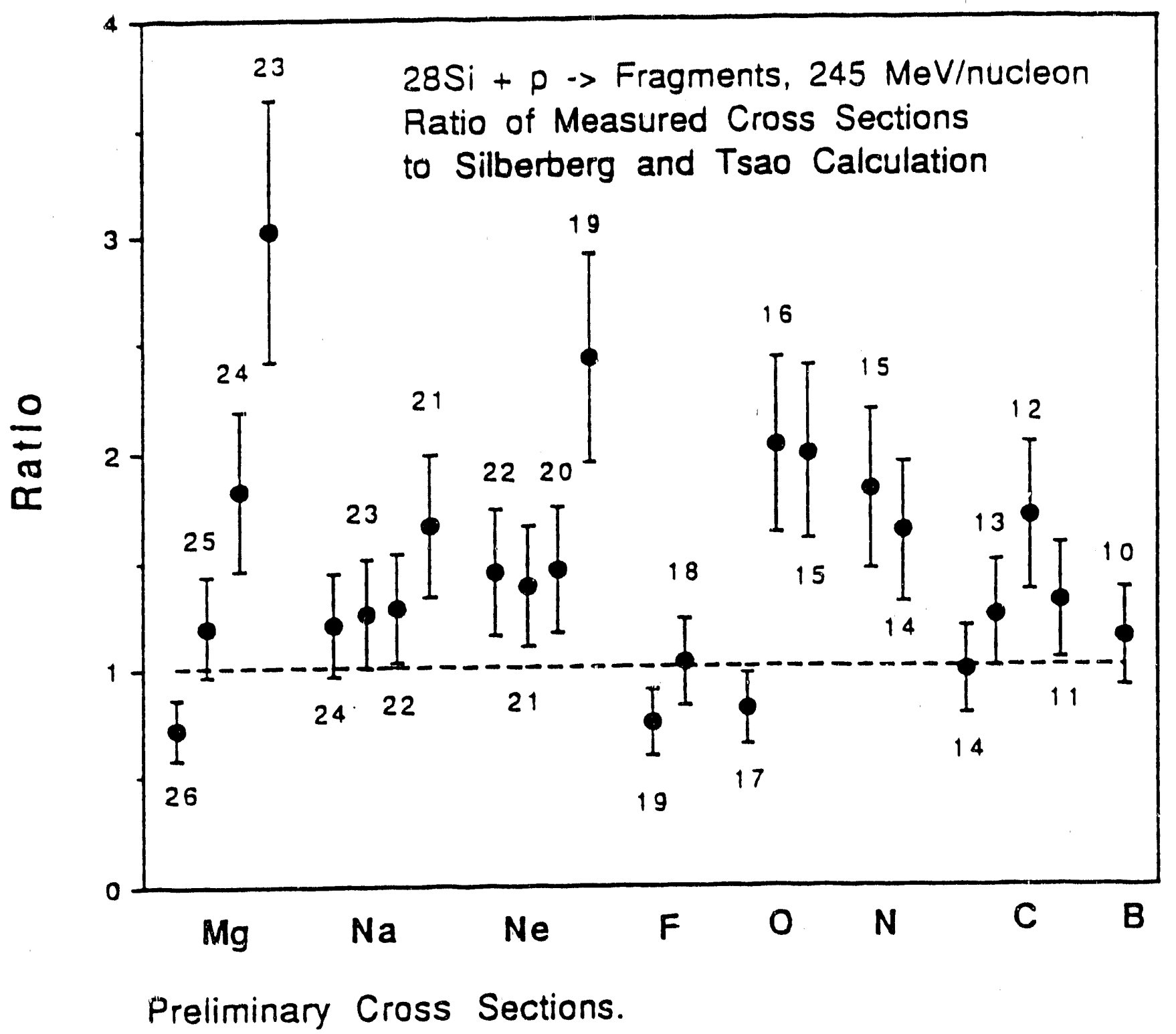

Fixed $20 \%$ error bars are shown only as an approximation.

Figure 17. Comparison of measured and predicted cross sections for fragments of ${ }^{28} \mathrm{Si}$ at $245 \mathrm{MeV} /$ nucleon. 

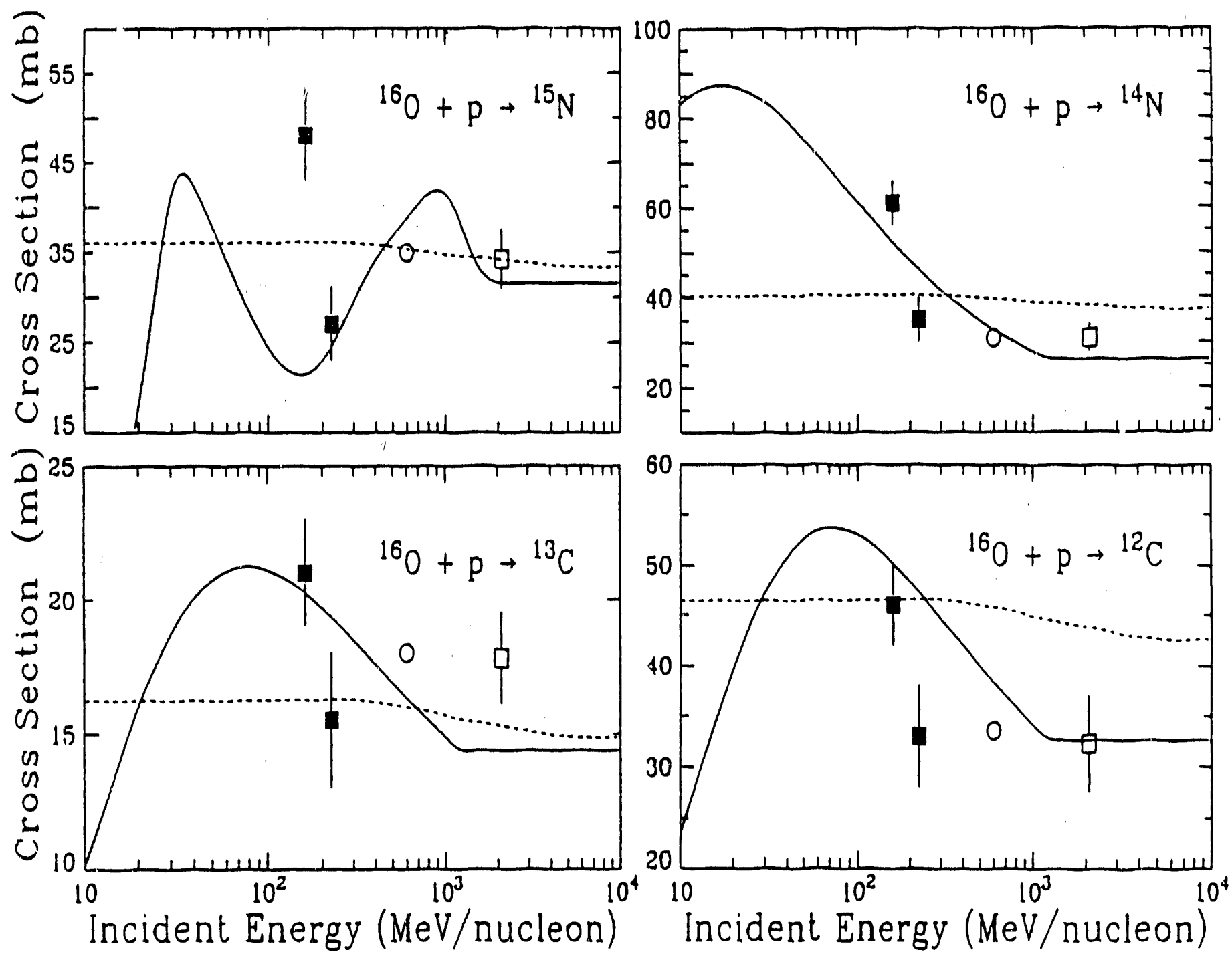

Figure 18. Comparison of 160 data at 160 and $225 \mathrm{MeV} /$ nucleon for $14,15 \mathrm{~N}$ and $12,13 \mathrm{C}$ with other measurements and with model predictions. 
What remains to be done on this dataset is to re-check the normalization and to derive the cross sections for the Aluminum isotopes. This latter has been a problem since there is still some beam contamination among the $\mathrm{Z}_{\mathrm{b}}-1$ fragments. A special analysis program is being developed to try to identify and remove this contamination.

Turning next to the 160 data, a backgıound removal technique has been developed and applied this past year. This resulted in considerably cleaner data, albeit with reduced statistics. Preliminary cross sections have been derived, and rcsults are shown for $14,15 \mathrm{~N}$ and ${ }^{13,12} \mathrm{C}$ in Figure 18, compared to other data and to predictions of the semi-empirical formulae (solid lines) and the parametric equations (dashed lines). Shown are our results at $160 \mathrm{MeV} /$ nucleon and our previous data from 160 at $225 \mathrm{MeV} /$ nucleon. (It should be noted here that the parametric equations were developed for data around $600 \mathrm{MeV} /$ nucleon, and the extrapolation to lower energies is probably not valid.) What is surprising is that our $160 \mathrm{MeV} /$ nucleon results give cross sections above previous results. Our values are consistent with the energy dependence predicted by the semi-empirical relations for ${ }^{14} \mathrm{~N},{ }^{12,13} \mathrm{C}$ (but not ${ }^{15} \mathrm{~N}$ ), but fall above the level predicted by the parametric equations (except for ${ }^{12} \mathrm{C}$ ). There is also a sharp rise in the cross sections between 225 and $160 \mathrm{MeV} /$ nucleon which calls into question one or the other of the datasets. (The proton stripping cross section for ${ }^{16} \mathrm{O}$ is particularly interesting since a high cross section here would call into question several previous analyses.)

What remains to be done here is to re-check the normalization and the background correction for each of the isotopes in the dataset, and to look at systematic effects between the 160 and 225 $\mathrm{MeV} /$ nucleon runs. In addition, we have a dataset at $370 \mathrm{MeV} /$ nucleon that is almost completed to the same level of analysis. Determining the cross sections at this energy will certainly shed some light on the validity of the lower energy points. 


\section{FUTURE PLANS}

In addition to completing the analysis of the datasets currently available, our long-range plans include obtairing new data through additional runs at HISS or an equivalent facility. Our current database, while extensive, is not complete. We have the hardware (in fact we began to use it in 4/91 before the MG set failed) to extend the measurements for the primary species to 1.6 $\mathrm{GeV} /$ nucleon. Further, we had planned for safety certification of a liquid Helium target and its use to obtain an equivalent database for interactions on LHe. Our technique is not limited to the iron peak but should be extendable, in a straight-forward manner, to isotopic measurement for elements in the range $\mathrm{Kr}-\mathrm{Zr}$ and, possibly, for the Xe-Ce region. These would be the first isotopic cross section measurements for beams heavier than the iron peak and would be important both for understanding the nuclear systematics and for interpreting astrophysical data.

We have also been urged to apply these techniques to other targets such as $\mathrm{O}, \mathrm{Al}, \mathrm{Mg}, \mathrm{Fe}$ or even $\mathrm{Pb}$. These datasets are needed for shielding applications and for understanding the radiobiological effects underneath complex shielding configurations. We can make the needed measurements!

With the original plan to phase out the Bevalac in FY95, we had planned to work with the EOS TPC group on a joint approach that would allow both groups to benefit from new runs. To that end we have been represented at EOS TPC group meetings and have begun discussions on detector interfaces and the like.

At the recent LBL PAC meeting (12/13/91) we presented the overall program as a Letter of Intent $(\mathrm{E} 1024 \mathrm{H})$. No allocation of beam time was requested silice that meeting focused on 1992 running, and the TPC will not be ready for a joint program in FY92. The letter from the PAC expressed support for the program and noted the successful runs and the apparent high quality of the data. They expressed willingness to consider beam time if a sponsor could be found for the Bevalac time.

The PAC's deliberations were conducted under the new time-line that envisions closure of the Bevalac after FY92, or possibly FY93. Their approach is to focus all available time on a few nuclear science programs such as the TPC. With such pressure for the TPC program to obtain meaningful data, it will be difficult to convince them of the need for joint running, however, we plan to try to maintain the dialog. Meanwhile, we are preserving the HISS detectors and the E$938 \mathrm{H}$ specific Hardware for a future eventuality.

A plan to continue Bevalac operations beyond FY92-93 under joint sponsorship of NASA, DoD and DOE was discussed at the meeting. We have also been trying to support such a plan with various people in Washington, DC. If some arrangement can be worked out for a longer life for the Bevalac, we would plan to be a part of the experimental program. We will be able, I believe, to maintain the team for the next few years working on the accumulated database. Thus, we should be in an excellent position to propose and utilize the Bevalac should extended operations become a reality. 

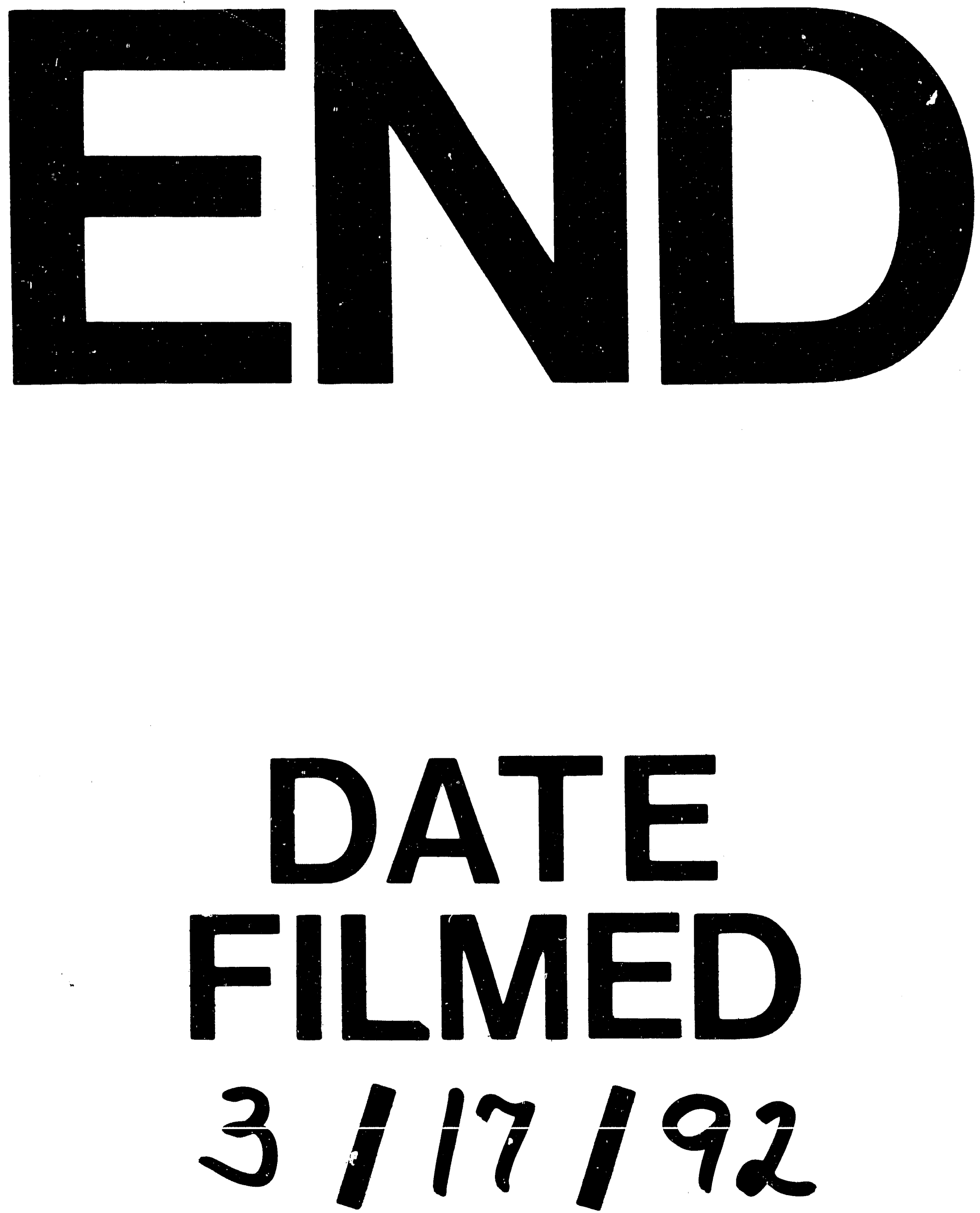


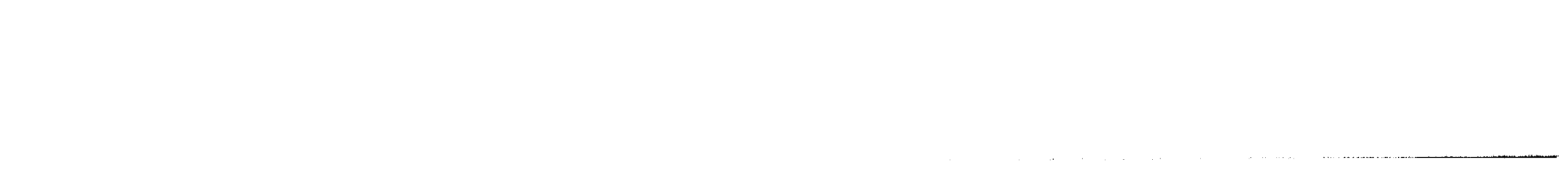

